\title{
1 RegVar: Tissue-specific Prioritization of Noncoding Regulatory
}

\section{Variants}

3 Hao Lu, Luyu Ma, Cheng Quan, Lei Li, Yiming Lu*, Gangqiao Zhou* and

4 Chenggang Zhang*

5

6 Beijing Institute of Radiation Medicine, State Key Laboratory of Proteomics, Beijing

7 100850, China.

8

$9 \quad$ * Corresponding authors.

10 E-mail: ylu.phd@gmail.com (Lu Y), zhougq114@126.com (Zhou G),

11 zhangcglab@gmail.com (Zhang C)

12

13 Running title: $\mathrm{Lu} H$ et al / Prioritization of Regulatory Variants

14

15 Article title letter count: 65

16 Running title letter count: 42

17 Abstract word count: 143

18 Total word count (from "Introduction" to "Discussion"): 4625

19 Total figures: 5

20 Total tables: 0

21 Total supplementary figures: 20

22 Total supplementary tables: 7

23 Total supplementary file: 1 


\section{Abstract}

26 Noncoding genomic variants constitute the majority of trait-associated genome

27 variations; however, identification of functional noncoding variants is still a challenge 28 in human genetics, and a method systematically assessing the impact of regulatory

29 variants on gene expression and linking them to potential target genes is still lacking.

30 Here we introduce a deep neural network (DNN)-based computational framework, 31 RegVar, that can accurately predict the tissue-specific impact of noncoding regulatory 32 variants on target genes. We show that, by robustly learning the genomic 33 characteristics of massive variant-gene expression associations in a variety of human 34 tissues, RegVar vastly surpasses all current noncoding variants prioritization methods 35 in predicting regulatory variants under different circumstances. The unique features of 36 RegVar make it an excellent framework for assessing the regulatory impact of any 37 variant on its putative target genes in a variety of tissues. RegVar is available as a 38 webserver at http://regvar.cbportal.org/.

39 KEYWORDS: Variant prioritization; Expression regulation; Deep neural network 40 


\section{Introduction}

43 Trait-associated genetic variants usually lie in noncoding genomic regions [1, 2], and 44 interpretation of functional noncoding variants is crucial for revealing the underlying genetic architecture and molecular mechanism of complex traits and diseases. Several methods have been developed to discriminate pathogenic variants from non-pathogenic ones using genomic sequences, functional annotations and evolutionary features, such as CADD [3], GWAVA [4], DeepSEA [5], LINSIGHT [6], etc. A common feature of these methods is that they focus on identifying rare pathogenic variants, which were thought to have stronger impact on human traits and diseases than common variants [7]. However, emerging evidences suggest that the majority of heritability for complex traits is likely to be explained by a substantial number of common regulatory variants with small additive effect sizes, in combination with a relatively smaller contribution from rare variants of moderate effect sizes [8-10]. Thus, a model that can distinguish both common and rare regulatory variants will provide new perspectives on the regulatory basis of complex traits.

Current pathogenic variant prioritization models are not suitable for identifying regulatory variants. A recent survey of existing methods for prioritizing noncoding variants showed that, although they achieved high precision in identifying pathogenic variants under certain circumstances, their performance in identifying regulatory variants was very poor [11]. This is because prioritization of regulatory variants is an even greater challenge than that of pathogenic ones. First, regulatory variants generally have weaker impact on gene expression compared to pathogenic ones, so it is more difficult to discriminate them from background, especially from adjacent non-functional variants sharing similar epigenetic marks. Second, it is challenging to

67 link regulatory variants to their target genes, which can be located far away from its regulator. Third, it is a challenge to establish tissue or cell type-specific models that can predict the regulatory impact of variants under different biological conditions. A 


\section{Materials and methods}

\section{Datasets}

number of methods have been proposed to predict the effects of regulatory variants in recent years [12-14], which, however, have their limitations in their application. For example, ExPecto relies on epigenetic marks at gene promoters to monitor the regulatory impact of variants on gene expression and thus could only assess promoter-proximal variants [12]; TIVAN connects various genomic features to expression quantitative trait loci (eQTLs) to estimate a variant's regulatory probability, but it was trained with promoter-proximal variants, which may introduce potential biases when applied to genome-wide variants prioritization [13]. Considering the vast majority of regulatory variants located far from the transcription start sites (TSSs) of target genes [15], a method that can robustly predict genome-wide regulatory variants as well as their potential target genes remains an urgent need.

Here we introduce a deep neural network (DNN)-based approach, RegVar, for the genome-wide assessment of the regulatory impact of noncoding variants on gene expression. RegVar has several key features: (i) it can predict both common and rare regulatory variants by learning their genomic characteristics from massive variant-gene associations in an unbiased manner; (ii) it predicts not only regulatory variants but their target genes by jointly learning the genomic patterns of both variants and genes and the chromatin interactions between them; (iii) it predicts the tissue-specific effects of variants by training models in multiple tissues with respective genomic patterns; and (iv) it can achieve excellent prediction accuracy by utilizing large training sets and deep learning algorithm. We show that RegVar outperforms existing prioritization methods in identifying regulatory variants and noncoding pathogenic variants from different backgrounds in various tissues. RegVar is available as a webserver at http://regvar.cbportal.org/. 
97 To construct the positive datasets, significant eVariant-eGene associations in 17

98 human tissues, which were also incorporated in the Roadmap Epigenomics projects

99 [2], were obtained from GTEx V7 release [16] (Figure 1 and Table S1).

100 Single-nucleotide variants (SNVs)-gene associations were selected and further filtered

101 by removing eVariants not marked by DNase I hypersensitive sites (DHSs)

102 annotations, which was demonstrated to be a key epigenetic marker of causal variants

103 [16]. Associations in sex chromosomes were also removed. For tissues of which the

104 numbers of significant associations exceed 100,000 (esophagus mucosa, lung, skeletal

105 muscle, and whole blood), we randomly selected 100,000 associations, as we found a

106 larger size cannot improve model performance (Figure S1). The final number of

107 positive associations for each tissue was shown in Table S1. For negative datasets,

108 four datasets were constructed, including: (i) random-variant set of shuffled

109 SNV-gene pairs where eVariants were replaced by random SNVs located $<=1 \mathrm{Mb}$

110 from the eGene TSS; (ii) mirrored-variant set of shuffled pairs where eVariants were

111 replaced by random SNVs located at similar distance (error $<=1 \mathrm{~kb}$ ) but the opposite

112 side of the eGene TSS; (iii) neighboring-variant set of shuffled pairs where eVariants

113 were replaced by random SNVs located adjacent $(<=1 \mathrm{~kb})$ to the positive ones; (iv)

114 random-gene set of shuffled SNV-gene pairs where eGenes were replaced by gene

115 TSSs located $<=1 \mathrm{Mb}$ of the eVariants. We selected a maximum distance at $1 \mathrm{Mb}$

116 between SNV and TSS in the datasets (i) and (iv), for it was observed that all positive

117 SNV-TSS pairs had a distance less than $1 \mathrm{Mb}$ (Figure S2). To determine the ratio

118 between positive and negative datasets, we assessed different ratios, including 1:1, 1:2,

$1191: 3,1: 5$, and 1:10, and found there was no significant difference of performances

120 among five models (Figure S3). Thus, we selected a ratio of 1:1 between the positive

121 and negative datasets to efficiently train the models. Variants in negative datasets

122 were selected from the dbSNP build 146 data after removing the shared variants

123 between GTEx and dbSNP datasets. Since eQTL variants are biased toward high

124 frequency variants (Figure S4), to ensure that our results were not influenced by the 
125 differences in minor allele frequency (MAF) between the positive variants and

126 negative controls, we defined additional sets of MAF-matched negative controls for

127 GTEx liver dataset, by the same strategy as the first three control datasets (i-iii)

128 described above.

129

\section{Annotation profiles}

131 We used three major categories of genomic profiles, including sequential, epigenetic

132 and evolutionary profiles (Table S2), to annotate our datasets using a customized

133 pipeline.

134

135 Sequential profiles

136 Sequential profiles consisted of 2-mer prefix and postfix and local 5-mer GC content

137 of SNV and TSS, SNV-caused transcription factor binding site (TFBS) affinity

138 changes, genomic distance between SNV and TSS and the orientations of SNV and

139 TSS.

140 To calculate TFBS affinity changes caused by variants, we obtained the position 141 frequency matrices of 602 TFs from the TRANSFAC [17] (523 TFs) and JASPAR

142 [18] (79 TFs) databases. TFMscan [19] was used to locate putative TFBS motifs by

143 scanning genomic DNA both forward and backward using these position frequency

144 matrices. A stringent threshold of $P$-value $<4.5 \mathrm{E}-5$ was used to determine significant

145 motifs. Variants located within these motifs were determined using BEDTools [20].

146 The TFBS affinity were calculated as described [21]. Specifically, the corrected

147 probabilities of observing a given nucleotide in a specific locus were calculated as

148 follows:

$149 \quad p(b, i)=\frac{f_{b, i}+s(b)}{N+\sum_{b^{\prime} \in\{A, T, C, G\}} s\left(b^{\prime}\right)}$ 
150 Where $b$ represents one specific base among $\mathrm{A}, \mathrm{T}, \mathrm{C}$, and $\mathrm{G}, i$ is the index of the site,

$151 f_{b, i}$ is the counts of base $b$ in site $i, N$ is the sum of counts of four bases, and $s(b)$ is the

152 pseudocount function. Here we assumed $s(b)$ to be $1 / 4$ for each of the four bases, then

$153 \sum_{b^{\prime} \in\{A, T, C, G\}} s\left(b^{\prime}\right)=1$

154 Hence, the corresponding PWM can be constructed as:

$155 \quad W(b, i)=\lg \frac{p(b, i)}{p(b)}$

156 where $p(b)$ is the background probability of base $b$ (assumed to be $1 / 4$ for four bases).

157 The TFBS affinity is calculated with

Affinity $=\sum_{i=1}^{w} W(b, i)$

159 where $w$ is the width of a PWM. We then calculated the average affinity change

160 between reference and alteration alleles as follows:

$\Delta$ Affinity $=\frac{\text { Affinity }_{A}-\text { Affinity }_{R}}{w}$

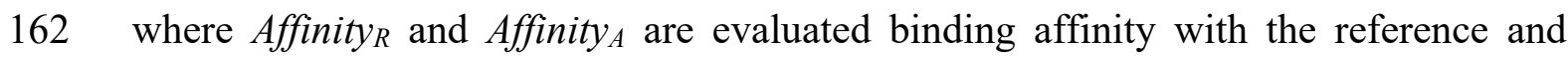

163 alteration alleles, respectively. Variants located within two or more TFBS motifs were

164 assigned with the $\Delta$ Affinity score with the maximum absolute value among all

165 AAffinity scores of the affected motifs and variants not located at any TFBS motif

166 were assigned a $\triangle$ Affinity score of 0.

Epigenetic profiles

169 Epigenetic profiles consisted of 31 histone modifications from the Roadmap

170 Epigenomics project [2], 25 chromatin states produced by ChromHMM [22], and

171 frequently interacted regions (FIREs) annotations from Hi-C study [23].

173 Evolutionary profiles

174 Evolutionary profiles consisted of vertebrate, placental mammal and primate phyloP

175 [24] and phastCons [25] scores based on the 46-way whole-genome alignment, and 
176 vertebrate phyloP and phastCons scores based on the 100-way whole-genome

177 alignment.

178 All annotations were expressed in genomic coordinates for the GRCh37/hg19 179 assembly of the human genome. Boolean variables were used to indicate if SNV or

180 TSS overlapped with chromatin marks (1) or not (0). For categorical annotations, all

181 n-level categorical values were first encoded to binary values and then converted to

182 several individual Boolean flags. For continuous annotations, feature values were

183 scaled to the range of $[0,1]$. More exactly, distance to TSS was scaled by

184 distance $=\min (1, \lg (\operatorname{abs}($ distance $)+1) / 6)$

185 phyloP scores scaled by

$186 \quad$ phyloP' $=\min (1, \operatorname{abs}($ phyloP $) / 5)$

187 and $\Delta$ Affinity scores scaled by

$188 \Delta$ Affinity' $=\min (1, \operatorname{abs}(\Delta$ Affinity $))$

\section{Model design and training}

191 We built a DNN-based classifier to model our dataset. The basic model in RegVar is a

192 fully connected neural network, in which each neuron in a layer receives inputs from

193 all outputs of the previous layer, except that the first layer receives inputs from the

194 original data matrix. Each layer in the network executes a linear transformation of the

195 corresponding inputs to integrate information from the previous layer, followed by a

196 non-linear transformation (namely the activation function) to rectify the linear result.

197 Here we employed three fully connected layers with 500, 200, and 60 units

198 respectively, and the most used rectified linear unit function (ReLU) as the activation

199 function. Exactly, one fully connected layer computes

$200 \quad$ output $=\operatorname{ReLU}(W X+b)$

201 where $X$ is the input, $W$ is the weight matrix, $b$ is the bias, and ReLU represents

202 rectified linear function

$203 \operatorname{ReLU}(x)=\max (0, x)$ 
204 The layer following the third fully connected layer is the final output layer to make

205 predictions about being a regulatory or non-regulatory variant on the specific gene,

206 with scaled probability ranging from 0 to 1 using

$207 \operatorname{sigmoid}(x)=\frac{1}{1+e^{-x}}$

208 To train the model, we selected the cross entropy loss function as the objective 209 function, which is defined as follows:

objective $=-\frac{1}{N} \sum_{i=1}^{N}\left[Y_{i} * \log \left(f\left(X_{i}\right)\right)+\left(1-Y_{i}\right) * \log \left(1-f\left(X_{i}\right)\right)\right]$

211 where $N$ is the number of samples in the training set, and $i$ is the index of each sample.

$212 Y_{i}$ and $X_{i}$ represent the $0 / 1$ label and the input features for sample $i$, respectively; and $213 f\left(X_{i}\right)$ represents the predicted probability output from the DNN model.

214 We conducted optimal search of hyper-parameters including the learning rate and 215 dropout proportion. Learning rates were set at 0.001, 0.005, and 0.01; dropout 216 proportions were set at $0,0.3$, and 0.5 . We selected the combinations of learning rates 217 and dropout proportions that achieved the highest prediction AUC in each of the four 218 models (Table S3-S6).

219 All training programs were written in Python language, using a deep neural 220 network implementation from the TensorFlow library.

\section{Model comparison}

223 We used the average receiver operating characteristic (ROC) curves computed from 224 10-fold cross-validation to evaluate model performances. Specifically, each dataset 225 comprising of the positive set and its negative counterpart was randomly split into a 226 training set and a testing set in a 9:1 ratio; the RegVar model was trained on the 227 training set and evaluated on the testing set. This process was repeated 10 times for each dataset, with independent sample split procedure each time. 
231 [26] and regBase [27] were used for model performance comparison in liver,

232 hippocampus and whole blood datasets. The random-variant, mirrored-variant and 233 neighboring-variant datasets were used for the evaluation, and random-gene dataset 234 was excluded as the existing methods didn't give prediction on potentially affected 235 genes. In addition, ExPecto was excluded from the random-variant and random-gene 236 models evaluation, because it focused on promoter-proximal variants thus resulted in 237 too few samples for the evaluation. For CADD, DeepSEA, and IW-scoring, we ran 238 the analysis using the corresponding online web services; for GWAVA, LINSIGHT, 239 ExPecto, TIVAN, and regBase, we downloaded the precomputed scores from the 240 corresponding source websites.

\section{Model external evaluation}

243 We downloaded liver eQTLs from the exSNP website [28], hippocampus eQTLs from 244 Schulz [29] and Ramasamy [30] eQTLs studies, and blood eQTLs from Westra [31] 245 eQTLs meta-analysis to evaluate performances of trained models. We identified all 246 SNV-TSS pairs and removed those overlapping with liver, hippocampus and whole 247 blood eQTLs in GTEx dataset. For negative controls, all SNVs in the external positive 248 datasets were removed from dbSNP build 146 and then four negative datasets were 249 constructed, as described above in model training, for each of the three independent 250 positive datasets. Also, the negative samples overlapping with the control sets used in 251 model training were further removed to avoid any valid set contamination. Then we 252 annotated each sample set with classifiers trained on GTEx eQTLs in the 253 corresponding tissue and compared classification results with ROC curves for the first 254 three sets with existing methods mentioned above.

255 Besides, we evaluated prediction capabilities of different methods on the liver and 256 blood eQTLs data from Brown eQTLs analysis [32], which have been used to test the 257 performance of TIVAN and regBase. We downloaded the compiled positive and 258 negative sets for Brown eQTLs data from the regBase website and compared 
259 performance of different methods on datasets in liver and blood (all testing datasets

260 are summarized in Table S7 and see Supplementary Methods for more details about 261 data processing).

\section{RegVar score distribution}

264 For each tissue, we trained an integrated RegVar model by pooling four negative 265 datasets to take all conditions together. 17 integrated RegVar models were applied to 266 annotate all possible SNV-gene pairs in chromosome 22. For each SNV in 267 chromosome 22, we obtained TSSs of all genes located within $1 \mathrm{Mb}$ of the variant 268 locus and combined the variant with each of these TSSs as a possible eQTL pair. 269 After mapped with all kinds of features, 65,844,726 sample pairs of 1,039,985 270 different SNVs were left and annotated with integrated RegVar models in 17 tissue 271 types. For each variant, the maximum annotated score of all its possible eQTL pairs 272 was set as the final RegVar score of the variant in each tissue. We next explored 273 distribution patterns of RegVar scores of all these variants.

\section{Tissue-shared/tissue-specific regulatory variants}

276 Stratified random sampling was performed to select 100,000 SNVs from 22 277 autosomes, and TSSs located within $1 \mathrm{Mb}$ of each variant locus were identified and 278 combined with the variant as a possible eQTL pair. After mapped with corresponding 279 features, 3,703,900 sample pairs were left. RegVar scores were obtained in 17 tissue 280 types and then converted to percentiles based on the corresponding merged training 281 sets to make results comparable across different tissues. For a particular variant, the 282 sample pair with the maximum percentile among all its possible eQTL pairs and 283 across 17 integrated models was set to be the final sample pair. We obtained 17 284 tissue-specific percentiles of all final sample pairs to form a percentile matrix. Then 285 K-means clustering, implemented by kmeans function in R language, was applied on 286 the matrix to get tissue-specific and tissue-shared regulatory variant clusters. Four 
287 tissue-specific epigenetic features, namely DHS, H3K4me1, H3K4me3, and H3K27ac,

288 were used to annotate these tissue-specific and tissue-shared regulatory variants.

290 Results

\section{Prioritization of regulatory variants in 17 human tissues}

292 To explore the influence of DHS filter on RegVar's prediction capability, we first 293 compared performance of models built from positive datasets without any filter, with 294 the DHS filter, with the ATAC-seq filter, and both positive and negative datasets with 295 the DHS filter in the liver dataset (Supplementary Methods), and found that models 296 built from the positive dataset with the DHS filter showed the most robust 297 performance in discriminating regulatory variants form different backgrounds (Figure 298 S5). We then utilized the DHS-filter-based RegVar to predict the tissue-specific 299 effects of genomic variants on gene expression in 17 human tissues. The averaged 300 ROC curves across 17 tissues showed that RegVar predicted regulatory variants and 301 their target genes with averaged AUCs of $0.965,0.917,0.693$, and 0.929 for the four 302 training datasets, respectively (Figure 2 and Table S1). This result demonstrated 303 RegVar could reliably discriminate positive regulatory variants from different 304 negative backgrounds. We then evaluated the performances of existing methods 305 CADD [3], GWAVA [4], DeepSEA [5], LINSIGHT [6], ExPecto [12], TIVAN [13], 306 IW-scoring [26], and regBase [27] on the same tasks. For CADD, we used C-scores. 307 For GWAVA, we used pathogenic scores with the corresponding control standards 308 (namely, unmatched, TSS, and region). For DeepSEA, we used eQTL-probability 309 scores. For IW-scoring, we used integrative scores without fitCons. For regBase, we 310 used regBase_Common prediction scores. For the three tested tissues: liver, 311 hippocampus, and whole blood, we found that only GWAVA, LINSIGHT, and 312 IW-scoring could make valid predictions with AUCs of 0.668-0.764 for 313 random-variant and 0.573-0.677 for mirrored-variant datasets, yet still much lower 314 than that of RegVar (0.957-0.969 for random-variant and 0.884-0.945 for 
315 mirrored-variant sets), while other five methods failed to show significant power in

316 distinguishing regulatory variants (Figure 3, Figure S6 and S7). For the

317 neighboring-variant set, which is more challenging, none of the existing methods

318 made valid predictions, compared to an AUC of $0.694-0.700$ for RegVar. We

319 additionally evaluate the prediction results of different methods by precision-recall

320 curves (PRC), and still RegVar showed the superior performance against other

321 methods (Figure S8, S9, and S10). After controlled for MAF, RegVar showed

322 comparable prediction capabilities in discriminating eQTLs from MAF-matched

323 benign variants, as demonstrated in liver samples (Figure S11), although with a slight

324 decrease in the neighboring-variant set, and the other eight methods still showed low 325 prediction capabilities as before.

326 To further confirm the results, we curated another three publicly available eQTL

327 datasets of liver, hippocampus, and whole blood, from the exSNP website [28], 328 Schulz [29] and Ramasamy [30] eQTL studies, and Westra [31] eQTL meta-analysis, 329 respectively, as independent testing sets. We found RegVar models trained with

330 GTEx datasets achieved almost equally accurate predictions in the three independent

331 testing sets, while all other methods still didn't show any obvious predictive powers in

332 the independent datasets (Figure 3, Figure S6-S10). To assess the robustness of

333 RegVar on imbalanced datasets, we then constructed independent validation sets for

334 liver eQTLs form the exSNP database with the sample ratio at 1:1, 1:2, 1:3, 1:5, and

335 1:10. We found that RegVar trained on GTEx datatsets at the 1:1 ratio showed robust

336 performance on both balanced and imbalanced datasets (Figure S12). In addition, we

337 also evaluated performance of different methods on the Brown eQTL data in liver and

338 blood, which have been used as testing data for regBase and TIVAN. Results showed

339 that in both tissues, RegVar trained on GTEx datasets showed comparable

340 performance (AUC $=0.858$ and 0.901 for liver and blood sets, respectively) with

341 regBase $(\mathrm{AUC}=0.883$ and 0.89 for liver and blood sets, respectively, equal to the

342 AUCs reported in the regBase paper. We also showed that RegVar models trained on 
343 the Brown eQTL data achieved even higher AUCs on both datasets (AUC $=0.952$

344 and 0.945 for liver and blood sets, respectively) compared with other methods (Figure 345 S13 and S14).

346 To investigate the robustness of RegVar on different settings of negative data 347 sampling in external evaluation, we constructed negative datasets for the exSNP 348 testing set by randomly selecting variants at wider genome regions, including: (i) 349 random-variant set comprising of random SNVs located $<=2$ and $5 \mathrm{Mb}$ from the 350 eGene TSS; (ii) mirrored-variant set comprising of random SNVs with a distance 351 error $<=2$ and $5 \mathrm{~kb}$; (iii) neighboring-variant set comprising of random SNVs located $352<=2$ and $5 \mathrm{~kb}$ to the positive ones; (iv) random-gene set comprising of random gene 353 TSSs located $<=2$ and $5 \mathrm{Mb}$ of the eVariants. RegVar models trained before 354 exhibited equal, or even slightly increased, prediction power in these independent 355 negative controls selected from wider genome regions, whereas other methods again 356 showed vary limited prediction performances (Figure S15). Altogether, these results 357 demonstrated the outstanding performance of RegVar on predicting regulatory impact 358 of noncoding variants.

359 We examined the feature importance of the four different models with Gini 360 impurity in liver (Supplementary Methods). For random-variant and mirrored-variant 361 models, the epigenetic patterns of variants were the most important feature sets, while 362 for the random-gene model, the epigenetic and sequential profiles of TSS were the 363 most important feature sets besides the distance between variant and TSS. Notably, 364 for the neighboring-variant model, evolutionary and sequential profiles of variants 365 became the most important feature sets (Figure S16). This is expected, as these 366 features could provide information of single-base resolution, which is crucial for 367 distinguishing regulatory variants from adjacent non-functional ones.

\section{RegVar score distribution}


370 We further compared performances of different models trained on the GTEx liver

371 eQTLs on each of negative datasets constructed for the independent exSNP testing set.

372 Results showed that AUCs of models from other types of negative data decreased by 373 0.031-0.133, 0.041-0.091, 0.062-0.105 for random-variant, mirrored-variant, and 374 neighboring-variant datasets, respectively, and that the integrated model obtained 375 superior capability in all testing datasets besides the models trained on the same type 376 of negative data (Figure S17). We then applied the integrated model to all SNVs in 377 chromosome $22(n=1,039,985)$ in 17 types of tissues and measured their regulatory 378 potentials with the corresponding RegVar scores. We calculated the optimal cutoff of 379 RegVar scores by maximizing the sum of specificity and sensitivity. We found that a 380 major proportion (84.5-94.0\%) of the DHS-supported eVariants were correctly 381 classified and a significant subset (24.6-39.1\%) of background variants were assigned 382 with RegVar scores above the cutoffs (Figure 4A and Figure S18). This result 383 suggests that a considerable portion of variants in human genome can function as 384 regulatory variants.

385 To further investigate the distribution of RegVar scores across different functional 386 genome regions, we mapped all annotated variants across 15 chromatin states 387 produced by ChromHMM [22] in liver and showed that variants at active/bivalent 388 promoters and enhancers usually have higher RegVar scores, while variants at 389 repressed and heterochromatin regions usually have lower scores (Figure 4C). This is 390 expected since most variants exert their effects through alteration of key regulatory 391 DNA elements [33]. Also, we observed a clear correlation between RegVar scores 392 and SNV-caused loss-of-function ( $\triangle$ Affinity $<=0$, ANOVA $F=422.6, P=0$ ) or 393 gain-of-function ( $\triangle$ Affinity $>=0$, ANOVA $F=23.62, P=5.52 \mathrm{E}-11$ ) of TFBSs

394 (Figure 4B), which means the extents of TFBS affinity alteration are positively 395 correlated with the probabilities of the causing variants to be functional. 
398 To evaluate the tissue specificity of the predicted regulatory variants, we applied the 399 integrated models in all 17 tissue types to randomly selected SNVs $(n=100,000)$ 400 across the human genome. K-means clustering of the RegVar score percentiles of 401 these SNVs identified 22 variant clusters, and one cluster was considered to be 402 enriched in a specific tissue if it was endowed with a K-means center percentile larger 403 than the percentile of the cutoff score in the corresponding tissue. We then identified 4048,11 , and 3 clusters of non-functional, tissue-specific regulatory, and tissue-shared 405 regulatory variants, from clusters which were enriched in $0,1-3$, and $>=12$ tissues 406 (there was no cluster enriched in 4-11 tissues), respectively (Figure 5A and Figure 407 S19). Using four epigenetic marks (DHS, H3K4me1, H3K27ac and H3K4me3) as 408 hallmarks of chromatin states, we showed that two clusters of tissue-shared variants 409 assigned with high RegVar scores (C6, C14) presented active promoter marks (DHS, $410 \mathrm{H} 3 \mathrm{~K} 4 \mathrm{me} 1, \mathrm{H} 3 \mathrm{~K} 27 \mathrm{ac}$, and H3K4me3) across all tissues, indicating they were enriched 411 at tissue-shared promoters. In contrast, the tissue-shared cluster assigned with 412 moderate RegVar scores (C2) presented active enhancer marks (DHS, H3K4me1, and $413 \mathrm{H} 3 \mathrm{~K} 27 \mathrm{ac})$ across all tissues, indicating their enrichment at tissue-shared enhancers.

414 We also found that most of the tissue-specific clusters presented active enhancer 415 marks specifically in the corresponding tissues, indicating their enrichment at 416 tissue-specific enhancers (Figure 5B). These results demonstrate the power of 417 RegVar in measuring the tissue-specific impact of regulatory variants.

\section{Prioritization of noncoding pathogenic variants in HGMD}

420 We further extended the framework of RegVar to prioritize noncoding pathogenic 421 variants. We used a simplified pathogenic RegVar model to learn the features of 422 noncoding pathogenic variants collected from the Human Gene Mutation database 423 (HGMD) [34]. We extracted disease-associated variants from the December 2016 424 release of HGMD public dataset. Small indels and variants overlapping any coding 425 sequence (as annotated in RefSeq genes from the UCSC Genome Browser) or 
426 essential splice sites (as annotated in GWAVA [4]) were filtered out. After mapping

427 remaining variants to all genomic annotations (Supplementary Methods), a final set of

4282078 disease-associated variants were used as the positive set of pathogenic 429 noncoding variants. For negative datasets, three datasets were constructed, including 430 (i) random-variant set of random SNVs sampled from the whole genome; (ii) 431 distance-control-variant set of random SNVs sampled from variants matched to the 432 pathogenic ones by the exact distance-to-nearest TSS (not necessarily near the same 433 TSSs as the pathogenic variants); (iii) neighboring-variant set of random SNVs 434 located $<=1 \mathrm{~kb}$ from the pathogenic ones. We selected a sample ratio at 1:10 due to 435 the small sample size of pathogenic variants, and negative variants overlapping any 436 coding sequence or essential splice sites were further filtered out. We then constructed 437 the pathogenic RegVar model on those different negative datasets. We found that 438 RegVar obtained superior capability in the random-variant and neighboring-variant 439 sets. The performances of regBase (AUC $=0.879)$, GWAVA (AUC $=0.874$ ), 440 IW-scoring $(\mathrm{AUC}=0.871)$ were comparable to RegVar's $(\mathrm{AUC}=0.885)$ in the 441 random-variant set, and regBase $(\mathrm{AUC}=0.704)$ was comparable to $\mathrm{RegVar}(\mathrm{AUC}=$ $4420.707)$ in the neighboring-variant set. In the distance-control-variant set, regBase 443 exhibited slight outperformance $(\mathrm{AUC}=0.845)$, followed by $\operatorname{RegVar}(\mathrm{AUC}=0.816)$ 444 and IW-scoring (AUC $=0.805)$ (Figure 6). These results demonstrated the 445 competence of the RegVar framework in discriminating between pathogenic and 446 benign variants.

447 We then explored the feature importance of the above three models 448 (Supplementary Methods). We found that sequential profiles were the most important 449 feature set in all three models, illustrating their prominent role in discriminating 450 noncoding pathogenic variants from different backgrounds; epigenetic and 451 evolutionary profiles were ranked second in the random-variant and 452 neighboring-variant models, respectively (Figure S20), which demonstrated their 
453 specific facility in separating noncoding pathogenic variants from a global and local

454 genome regions, respectively.

456 Discussion

457 Noncoding variants play a prominent role in many diseases and complex traits 458 through various intricate mechanisms [35, 36]. Nevertheless, variants would exert 459 their effects by affecting the expression of specific genes. It is a great challenge to 460 link regulatory variants, especially in long distance, to their target genes. Here we 461 show that through jointly learning the genomic patterns of variants and genes, RegVar 462 provides helpful information for mapping regulatory variants to their target genes. We expect RegVar can contribute to current limited understanding of genetic architecture 464 of human genome and help to uncover novel molecular mechanisms underlying 465 complex traits and diseases.

466 A number of methods have been developed for measuring the consequence and 467 importance of noncoding variants. Though differing from each other in the underlying 468 intuitions and specific algorithm frameworks, they mainly focused on predicting the 469 pathogenic effect of variants. Therefore a vast number of noncoding variants with 470 smaller regulatory effects would be neglected. Here we demonstrated the unique 471 ability of RegVar to prioritize regulatory variants against different backgrounds. We 472 found that in the random-variant, mirrored-variant, and random-gene datasets, 473 RegVar obtained accurate and robust prediction capability; in the neighboring-variant 474 dataset, RegVar exhibited relatively weak prediction power, but still superior to 475 existing methods. These results demonstrate RegVar as an integrated model to 476 identify genome-wide regulatory variants, and it may be not suitable for fine-mapping 477 studies in limited regions. Applying RegVar to all SNVs in chromosome 22, we show 478 that there is a considerable portion of variants across the wide genome showing large 479 probabilities with which to regulate the expression of certain target genes. The reason 
bioRxiv preprint doi: https://doi.org/10.1101/2021.04.17.440295; this version posted April 19, 2021. The copyright holder for this preprint (which was not certified by peer review) is the author/funder, who has granted bioRxiv a license to display the preprint in perpetuity. It is made available under aCC-BY-NC-ND 4.0 International license.

480 they have not been reported may be that their effects are too subtle to be detected,

481 coupled with limited sample sizes and low statistical power.

482 


\section{Code availability}

484 The RegVar online server is freely available at http://regvar.cbportal.org/.

485 Downloadable datasets and source code to run RegVar on local personal computers 486 and scripts to generate figures in the manuscript are also provided at the RegVar 487 website.

488

489 CRediT author statement

490 Hao Lu: Methodology, Investigation, Software, Visualization, Writing - Original 491 Draft, Writing - Review \& Editing. Luyu Ma: Methodology, Investigation, 492 Visualization. Cheng Quan: Investigation, Software. Lei Li: Methodology, 493 Investigation, Visualization. Yiming Lu: Conceptualization, Methodology, 494 Investigation, Visualization, Writing - Original Draft, Writing - Review \& Editing. 495 Gangqiao Zhou: Conceptualization, Supervision. Chenggang Zhang: 496 Conceptualization, Supervision. All authors read and approved the final manuscript.

499 Competing interests

500 The authors have declared no competing interests.

501

\section{Acknowledgements}

503 This work was supported by the General Program of the National Natural Science 504 Foundation of China (Grant No. 31771397) and the Beijing Nova Program (Grant No. 505 20180059).

507 Authors' ORCID IDs

508 0000-0002-8157-4158 (Hao Lu)

509 0000-0003-2907-8410 (Luyu Ma)

510 0000-0003-1859-9683 (Cheng Quan) 
bioRxiv preprint doi: https://doi.org/10.1101/2021.04.17.440295; this version posted April 19, 2021. The copyright holder for this preprint (which was not certified by peer review) is the author/funder, who has granted bioRxiv a license to display the preprint in perpetuity. It is made available under aCC-BY-NC-ND 4.0 International license.

511 0000-0002-5100-2124 (Lei Li)

512 0000-0001-8005-2705 (Yiming Lu)

513 0000-0002-4895-5063 (Gangqiao Zhou)

514 0000-0002-4521-3304 (Chenggang Zhang)

515

516 


\section{References}

518 [1] Maurano MT, Humbert R, Rynes E, Thurman RE, Haugen E, Wang H, et al.

519 Systematic localization of common disease-associated variation in regulatory DNA.

520 Science 2012;337:1190-5.

521 [2] Kundaje A, Meuleman W, Ernst J, Bilenky M, Yen A, Heravi-Moussavi A, et al.

522 Integrative analysis of 111 reference human epigenomes. Nature 2015;518:317-30.

523 [3] Kircher M, Witten DM, Jain P, O'Roak BJ, Cooper GM, Shendure J. A general

524 framework for estimating the relative pathogenicity of human genetic variants. Nat

525 Genet 2014;46:310-5.

526 [4] Ritchie GR, Dunham I, Zeggini E, Flicek P. Functional annotation of noncoding 527 sequence variants. Nat Methods 2014;11:294-6.

528 [5] Zhou J, Troyanskaya OG. Predicting effects of noncoding variants with deep 529 learning-based sequence model. Nat Methods 2015;12:931-4.

530 [6] Huang Y-F, Gulko B, Siepel A. Fast, scalable prediction of deleterious noncoding 531 variants from functional and population genomic data. Nat Genet 2017;49:618-24.

532 [7] Zeng Y, Wang G, Yang E, Ji G, Brinkmeyer-Langford CL, Cai JJ. Aberrant gene 533 expression in humans. PLoS Genet 2015;11:e1004942.

534 [8] Torkamani A, Wineinger NE, Topol EJ. The personal and clinical utility of 535 polygenic risk scores. Nat Rev Genet 2018;19:581-90.

536 [9] Visscher PM, Wray NR, Zhang Q, Sklar P, McCarthy MI, Brown MA, et al. 10 537 years of GWAS discovery: biology, function, and translation. Am J Hum Genet $538 \quad 2017 ; 101: 5-22$.

539 [10] Fuchsberger C, Flannick J, Teslovich TM, Mahajan A, Agarwala V, Gaulton KJ,

540 et al. The genetic architecture of type 2 diabetes. Nature 2016;536:41-7.

541 [11] Liu L, Sanderford MD, Patel R, Chandrashekar P, Gibson G, Kumar S.

542 Biological relevance of computationally predicted pathogenicity of noncoding 543 variants. Nat Commun 2019;10:330.

544 [12] Zhou J, Theesfeld CL, Yao K, Chen KM, Wong AK, Troyanskaya OG. Deep 545 learning sequence-based ab initio prediction of variant effects on expression and 546 disease risk. Nat Genet 2018;50:1171-9.

547 [13] Chen L, Wang Y, Yao B, Mitra A, Wang X, Qin X. TIVAN: tissue-specific 548 cis-eQTL single nucleotide variant annotation and prediction. Bioinformatics $549 \quad 2019 ; 35: 1573-5$.

550 [14] Li MJ, Li M, Liu Z, Yan B, Pan Z, Huang D, et al. cepip: context-dependent 551 epigenomic weighting for prioritization of regulatory variants and disease-associated 552 genes. Genome Biol 2017;18:52.

553 [15] Tewhey R, Kotliar D, Park DS, Liu B, Winnicki S, Reilly SK, et al. Direct 554 identification of hundreds of expression-modulating variants using a multiplexed 555 reporter assay. Cell 2016;165:1519-29.

556 [16] Consortium G. Genetic effects on gene expression across human tissues. Nature 557 2017;550:204-13. 
558 [17] Matys V, Fricke E, Geffers R, Gössling E, Haubrock M, Hehl R, et al. 559 TRANSFAC $\AA$ : transcriptional regulation, from patterns to profiles. Nucleic Acids $560 \quad$ Res 2003;31:374-8.

561 [18] Mathelier A, Fornes O, Arenillas DJ, Chen C-y, Denay G, Lee J, et al. JASPAR 562 2016: a major expansion and update of the open-access database of transcription 563 factor binding profiles. Nucleic Acids Res 2015;44:D110-D5.

564 [19] Liefooghe A, Touzet H, Varré J-S. Large scale matching for position weight 565 matrices. Annual Symposium on Combinatorial Pattern Matching 2006:401-12.

566 [20] Quinlan AR, Hall IM. BEDTools: a flexible suite of utilities for comparing 567 genomic features. Bioinformatics 2010;26:841-2.

568 [21] Wasserman WW, Sandelin A. Applied bioinformatics for the identification of 569 regulatory elements. Nat Rev Genet 2004;5:276-87.

570 [22] Ernst J, Kellis M. ChromHMM: automating chromatin-state discovery and 571 characterization. Nat Methods 2012;9:215-6.

572 [23] Schmitt AD, Hu M, Jung I, Xu Z, Qiu Y, Tan CL, et al. A compendium of 573 chromatin contact maps reveals spatially active regions in the human genome. Cell 574 Rep 2016;17:2042-59.

575 [24] Pollard KS, Hubisz MJ, Rosenbloom KR, Siepel A. Detection of nonneutral 576 substitution rates on mammalian phylogenies. Genome Res 2010;20:110-21.

577 [25] Siepel A, Bejerano G, Pedersen JS, Hinrichs AS, Hou M, Rosenbloom K, et al. 578 Evolutionarily conserved elements in vertebrate, insect, worm, and yeast genomes. 579 Genome Res 2005;15:1034-50.

580 [26] Wang J, Dayem Ullah AZ, Chelala C. IW-Scoring: an Integrative Weighted 581 Scoring framework for annotating and prioritizing genetic variations in the noncoding 582 genome. Nucleic Acids Res 2018;46:e47.

583 [27] Zhang S, He Y, Liu H, Zhai H, Huang D, Yi X, et al. regBase: whole genome 584 base-wise aggregation and functional prediction for human non-coding regulatory 585 variants. Nucleic Acids Res 2019;47:e134.

586 [28] Yu C-H, Pal LR, Moult J. Consensus genome-wide expression quantitative trait 587 loci and their relationship with human complex trait disease. OMICS 2016;20:400-14. 588 [29] Schulz H, Ruppert A-K, Herms S, Wolf C, Mirza-Schreiber N, Stegle O, et al. 589 Genome-wide mapping of genetic determinants influencing DNA methylation and 590 gene expression in human hippocampus. Nat Commun 2017;8:1511.

591 [30] Ramasamy A, Trabzuni D, Guelfi S, Varghese V, Smith C, Walker R, et al. 592 Genetic variability in the regulation of gene expression in ten regions of the human 593 brain. Nat Neurosci 2014;17:1418-28.

594 [31] Westra H-J, Peters MJ, Esko T, Yaghootkar H, Schurmann C, Kettunen J, et al. 595 Systematic identification of trans eQTLs as putative drivers of known disease 596 associations. Nat Genet 2013;45:1238-43.

597 [32] Brown CD, Mangravite LM, Engelhardt BE. Integrative modeling of eQTLs and 598 cis-regulatory elements suggests mechanisms underlying cell type specificity of 599 eQTLs. PLoS Genet 2013;9:e1003649. 
600 [33] Lee PH, Lee C, Li X, Wee B, Dwivedi T, Daly M. Principles and methods of 601 in-silico prioritization of non-coding regulatory variants. Hum Genet 2018;137:15-30.

602 [34] Stenson PD, Mort M, Ball EV, Evans K, Hayden M, Heywood S, et al. The 603 Human Gene Mutation Database: towards a comprehensive repository of inherited 604 mutation data for medical research, genetic diagnosis and next-generation sequencing 605 studies. Hum Genet 2017;136:665-77.

606 [35] Albert FW, Kruglyak L. The role of regulatory variation in complex traits and 607 disease. Nat Rev Genet 2015;16:197-212.

608 [36] Khurana E, Fu Y, Chakravarty D, Demichelis F, Rubin MA, Gerstein M. Role of 609 non-coding sequence variants in cancer. Nat Rev Genet 2016;17:93-108.

610

611 


\section{Figure legends}

613 Figure 1 A flowchart showing the workflow of RegVar

614

615 Figure 2 Average ROC curves for 10-fold cross-validation experiments of 616 RegVar

617 For each of the four training sets, the ROC curves are averaged across 17 human 618 tissues. Error bars represent the standard deviation averaged over tissues.

620 Figure 3 ROC curves of nine computational methods distinguishing regulatory variants from different backgrounds in liver

Results are shown for ROC curves from 10-fold cross-validation experiments in the

623 GTEx liver eQTL dataset (top) and from external evaluation experiments in the 624 exSNP liver eQTL dataset (bottom). Negative datasets were from either random 625 selected variants (random-variant sets) (left), matched variants by distance but at the 626 opposite side of the eGene TSS (mirrored-variant sets) (middle), or neighboring 627 variants located adjacent $(<=1 \mathrm{~kb})$ to the positive ones (neighboring-variant sets) 628 (right). Negative datasets from random selected TSSs (random-gene sets) are not 629 shown since other existing methods didn't give prediction on potentially affected 630 genes. Any overlap between the exSNP liver eQTLs and GTEx eQTLs and overlap 631 between their corresponding negative sets were removed. ExPecto results are not 632 shown for random-variant sets because it resulted in too few samples for ROC curve 633 analysis.

634

635 Figure 4 RegVar scores across all variants in chromosome 22 annotated in the 636 integrated liver RegVar model

637 A. Histogram showing the RegVar scores distribution across all SNVs in chromosome $63822(N=1,039,985)$ (lightblue) and SNVs in GTEx liver eQTLs (orange). Dashed line 639 indicates the optimal cutoff score in liver training set. Numbers of variants blow or 
640 above the cutoff score are embedded. B. Spine plot showing the correlation between

641 RegVar scores and SNV-caused TFBS affinity changes. C. Violin plots showing the

642 RegVar score distributions across 15 chromatin states (BivFlnk, flanking bivalent

643 TSS/enhancers; Enh, enhancers; EnhBiv, bivalent enhancers; EnhG, genic enhancers;

644 Het, heterochromatin; ReprPC, repressed PolyComb; ReprPCWk, weak repressed

645 PolyComb; Quies, quiescent/low; TssA, active TSS; TssAFlnk, flanking active TSS;

646 TssBiv, bivalent/poised TSS; Tx, strong transcription; TxFlnk, transcription at gene 5'

647 and 3'; TxWk, weak transcription; ZNF/Rpts, ZNF genes \& repeats). Embedded

648 boxplots indicate medians (center bars), and the first and third quartiles (lower and

649 upper hinges).

650

651 Figure 5 Tissue-shared and tissue-specific regulatory variants and 652 non-functional variants identified in K-means clustering

653 A. RegVar score percentiles for different clusters of variants $(N=100,000)$ annotated 654 with the integrated RegVar models in 17 tissues (ACC, anterior cingulate cortex; AG, 655 adrenal gland; AO, aorta; EM, esophagus mucosa; HI, hippocampus; LI, liver; LU, 656 lung; LV, left ventricle; OV, ovary; PA, pancreas; SC, sigmoid colon; SI, small 657 intestine; SM, skeletal muscle; SN, substantia nigra; SP, spleen; ST, stomach; WB, 658 whole blood). B. Enrichment proportion of different clusters of variants in genome 659 regions with four epigenomic annotations (DHS, H3K4me1, H3K4me3, and H3K27ac) 660 in 10 selected tissues.

661

662 Figure 6 ROC curves of seven computational methods distinguishing noncoding 663 pathogenic variants from different backgrounds

664 Positive samples were from the of HGMD noncoding pathogenic variants $(N=2078)$.

665 Negative samples were from either random selected variants (random-variant set) 666 (left), matched variants by the exact distance-to-nearest TSS (not necessarily near the 667 same TSS as each pathogenic variant) (distance-control-variant set) (middle), or 
668 neighboring variants located adjacent $(<=1 \mathrm{~kb})$ to the positive ones

669 (neighboring-variant set) (right). Because GWAVA was trained on the HGMD

670 noncoding mutations, we filtered out GWAVA training positive-variants in evaluating

671 its performance. TIVAN and ExPecto results are not shown because they only

672 provides tissue-specific regulatory variants prioritization scores.

673

674 Supplementary material

675 Figure S1 AUCs of RegVar models with different sample sizes

676 Positive samples were from the GTEx skeletal muscle eQTLs. Sample sizes were set

677 from 20,000 to 180,000 , step by 20,000 , and the full sample size $(N=204,124)$.

678

679 Figure S2 Histogram showing the distance between variant loci and TSS

680 Results are shown for samples from the GTEx eQTLs from 17 tissues (A-Q).

681

682 Figure S3 ROC curves for 10-fold cross-validation experiments at different 683 ample ratios between positive and negative datasets

684 Positive samples were from the GTEx liver eQTLs. Negative samples were selected at 685 the ratio of $1: 1,1: 2,1: 3,1: 5$, and $1: 10$ between positive $(\mathrm{P})$ and negative $(\mathrm{N})$ datasets.

686

687 Figure S4 MAF distribution (A) and common and rare variant proportion (B) in 688 dbSNP variants and GTEx eQTLs

689

690 Figure S5 ROC curves of RegVar models trained on samples with different

691 filters

692 Results are shown for models trained on the original GTEx liver eQTLs $(N=268,673)$

693 with all feature sets as predictors and with DHS peaks alone as a predictor, RegVar 694 models trained on the GTEx liver eQTLs filtered by DHS peaks $(N=41,636)$ and 
695 filtered by ATAC profiles $(N=3731)$, and RegVar models trained on both the GTEx

696 liver eQTLs and controls filtered by DHS peaks.

697

698 Figure S6 ROC curves of nine computational methods distinguishing regulatory

699 variants from different backgrounds in hippocampus

700 Results are shown for ROC curves from 10-fold cross-validation experiments in the

701 GTEx hippocampus eQTL dataset (top) and from external evaluation experiments in

702 Schulz and Ramasamy hippocampus eQTL dataset (bottom). Plots are similar to

703 Figure 3.

704

705

Figure S7 ROC curves of nine computational methods distinguishing regulatory

variants from different backgrounds in whole blood

Results are shown for ROC curves from 10-fold cross-validation experiments in the

GTEx whole blood eQTL dataset (top) and from external evaluation experiments in

Westra whole blood eQTL dataset (bottom). Plots are similar to Figure 3.

711 Figure S8 PRC curves of nine computational methods distinguishing regulatory variants from different backgrounds in liver

713 Results are shown for PRC curves for the same result as Figure 3.

Figure S9 PRC curves of nine computational methods distinguishing regulatory variants from different backgrounds in hippocampus

717 Results are shown for PRC curves for the same result as Supplementary Figure S6

Figure S10 PRC curves of nine computational methods distinguishing regulatory

721 Results are shown for PRC curves for the same result as Supplementary Figure S7 
723 Figure S11 ROC curves of nine computational methods distinguishing regulatory

724 variants in liver from MAF-matched variants

725 Positive samples were from the GTEx liver eQTLs $(N=41,636)$. Negative samples in

726 three control datasets were randomly selected from MAF-matched variants to the 727 positive ones. Plots are similar to Figure 3.

728

Figure S12 ROC curves of RegVar distinguishing regulatory variants from

730 different backgrounds in liver at different sample ratio

731 Positive samples were from the exSNP liver eQTL dataset. Negative samples were

732 selected at the ratio of $1: 1,1: 2,1: 3,1: 5$, and 1:10 between positive $(\mathrm{P})$ and negative

$733(\mathrm{~N})$ datasets.

734

735 Figure S13 ROC curves of nine computational methods distinguishing regulatory 736 variants in Brown liver eQTLs

737 Positive and negative samples were from the Brown liver eQTLs complied by 738 regBase.

740 Figure S14 ROC curves of nine computational methods distinguishing regulatory 741 variants in Brown blood eQTLs

742 Positive and negative samples were from the Brown blood eQTLs complied by 743 regBase.

745 Figure S15 ROC curves of nine computational methods distinguishing regulatory

746 variants in the exSNP liver eQTLs from different backgrounds

747 Positive samples were from the exSNP liver eQTL dataset $(N=4307)$. Negative 748 samples in three control datasets were randomly selected from wider genome regions:

749 (i) random-variant set comprising of random SNVs located $<=2$ (Top) and 5 (bottom)

$750 \mathrm{Mb}$ from the eGene TSS; (ii) mirrored-variant set comprising of random SNVs with a 
751 distance error $<=2$ (Top) and 5 (bottom) kb; (iii) neighboring-variant set comprising

752 of random SNVs located $<=2$ (Top) and 5 (bottom) kb to the positive ones. Result for

753 random-gene set comprising of random gene TSSs located $<=2$ and $5 \mathrm{Mb}$ of the

754 eVariants was not shown since other existing methods didn't give prediction on

755 potentially affected genes.

756

757 Figure S16 Barplots showing the relative Gini importance for different models

758 Results are shown for models trained on random-variant (A), mirrored-variant (B),

759 neighboring-variant $(\mathbf{C})$, and random-gene (D) datasets of the GTEx liver eQTLs ( $N$

$760=41,636)$. All features were divided into seven groups: distance between variant and

761 TSS (Distance), sequential profiles of variant (VarSeq) and TSS (TSSSeq), epigenetic

762 profiles of variant (VarEpi) and TSS (TSSEpi), evolutionary profiles of variant

763 (VarEvo) and TSS (TSSEvo). Error bars represents the standard error averaged over

76410 times.

765

Figure S17 ROC curves of different RegVar models distinguishing regulatory variants in the exSNP liver eQTLs from each of the negative datasets

Positive samples were from the exSNP liver eQTLs $(N=4307)$. Models based on the random-gene dataset was not evaluated in this analysis, because it aimed to identify potential eGenes for each eVariant and wasn't suitable for prioritization of positive variants.

773 Figure S18 Histogram showing the RegVar scores distribution

774 Results are shown for all SNVs in chromosome 22 (lightblue) and SNVs in the GTEx eQTLs (orange). Variants were annotated in integrated RegVar models in 16 tissues

776 (A-P). Dashed line indicates the optimal cutoff scores in the corresponding training set. Numbers of variants blow or above the cutoff scores are embedded. 
779 Figure S19 Identification of tissue-shared/tissue-specific cluster of variants

780 An orange cell indicate the cluster was endowed with an K-means center percentile

781 larger than the percentile of the RegVar cutoff score in the corresponding tissue, and 782 an skyblue cell if not. ACC, anterior cingulate cortex; AG, adrenal gland; AO, aorta;

783 EM, esophagus mucosa; HI, hippocampus; LI, liver; LU, lung; LV, left ventricle; OV, 784 ovary; PA, pancreas; SC, sigmoid colon; SI, small intestine; SM, skeletal muscle; SN, 785 substantia nigra; SP, spleen; ST, stomach; WB, whole blood.

787 Figure S20 Barplots showing the relative Gini importance for different 788 pathogenic RegVar models

789 Results are shown for models trained on random-variant (A), distance-control-variant

790 (B), and neighboring-variant (C) datasets of HGMD noncoding pathogenic variants $791(N=2,078)$. All features were divided into four groups: distance to the nearest TSS 792 (Distance), and sequential (Seq), epigenetic (Epi), and evolutionary (Evo) profiles of 793 the variant. Error bars represents the standard error averaged over 10 times.

Table S1 Sample sizes and AUCs of the 4 training sets in 17 tissues

Table S2 Summary of genomic features used by RegVar

Table S3 AUCs under different learning rates and dropout proportions in the liver random-variant set

Table S4 AUCs under different learning rates and dropout proportions in the liver mirrored-variant set

Table S5 AUCs under different learning rates and dropout proportions in the 
bioRxiv preprint doi: https://doi org/10.1101/2021.0417.440295; this version posted April 19 2021. The copyright holder for this preprint (which was not certified by peer review) is the author/funder, who has granted bioRxiv a license to display the preprint in perpetuity. It is made available under aCC-BY-NC-ND 4.0 International license.

807

808 Table S6 AUCs under different learning rates and dropout proportions in the 809 liver random-gene set

810

811 Table S7 Testing sets used for evaluation of RegVar and other methods

812

813 File S1 Supplementary methods

814 


\section{Dataset}

Genomic profiles

Model

\begin{tabular}{|} 
Positive dataset \\
\hline GTEx SNV-TSS \\
\hline Negative dataset \\
\hline Random-variant set \\
\hline Mirrored-variant set \\
\hline Neighboring-variant set \\
\hline Random-gene set \\
\hline
\end{tabular}

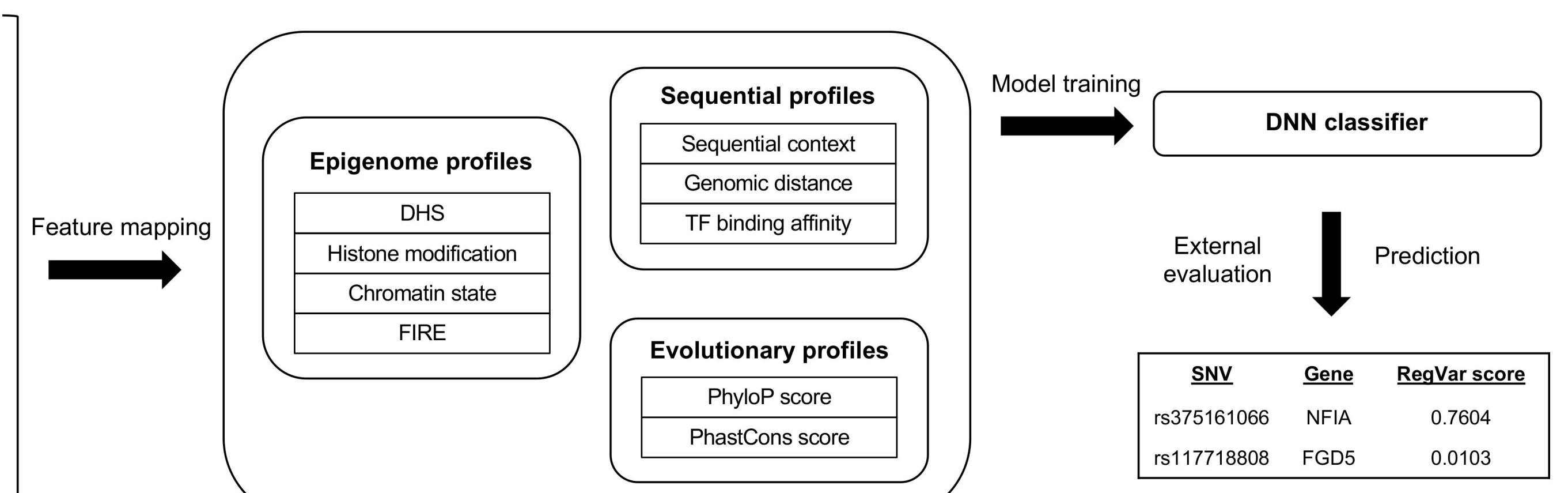




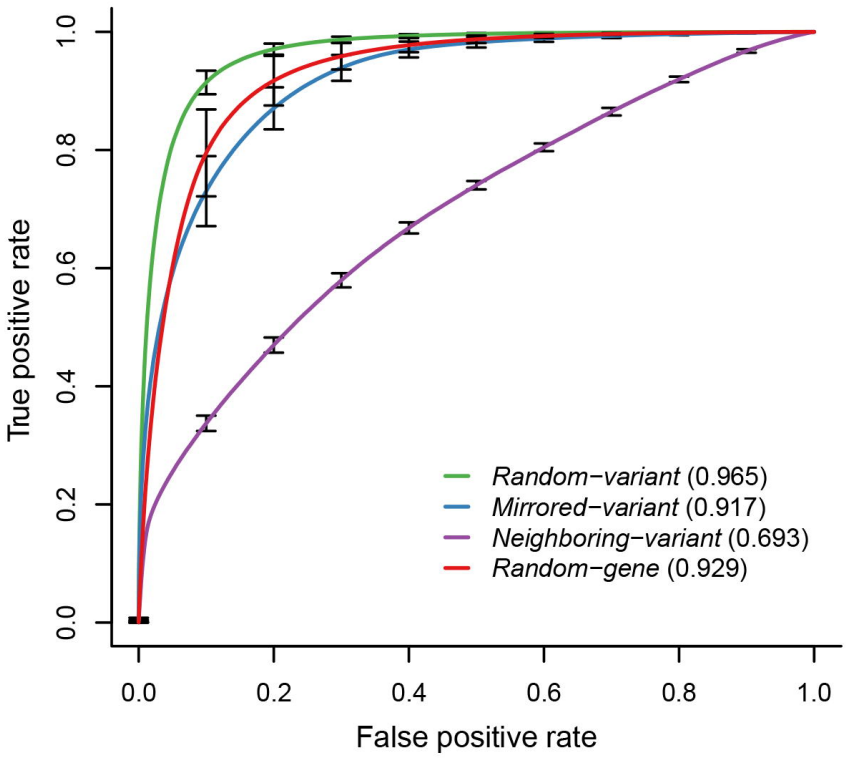


Random-variant

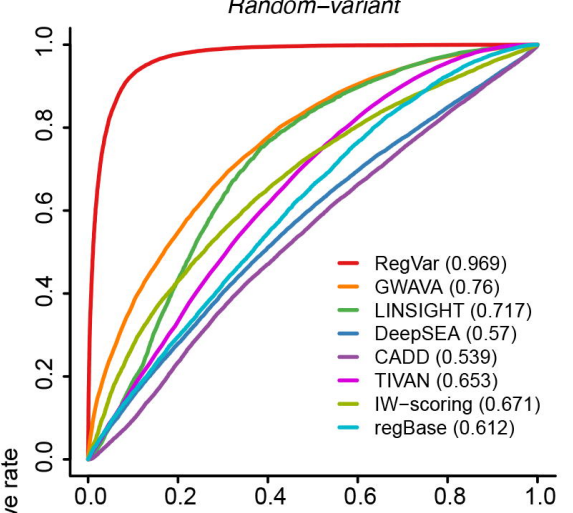

Mirrored-variant

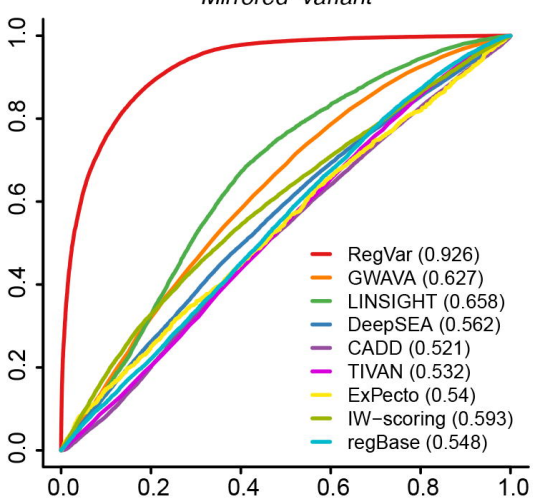

Neighboring-variant

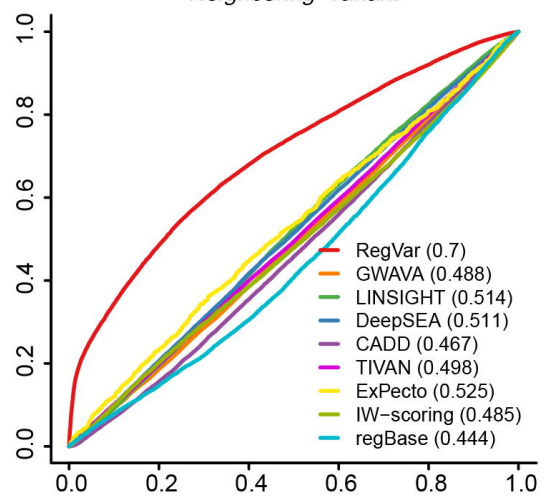

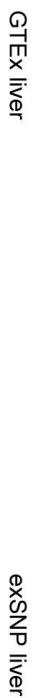

- RegVar (0.97)

- GWAVA (0.759)

- DeepSEA (0.619)

- CADD (0.536)

- TIVAN (0.699)

- IW-scoring $(0.658)$

- regBase (0.619)

웅.

พ

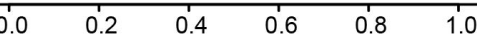

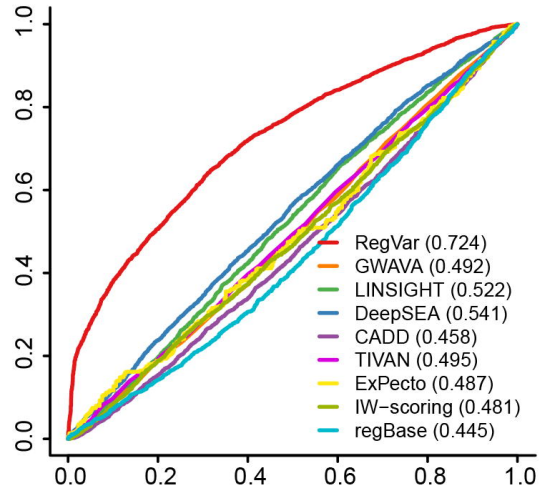



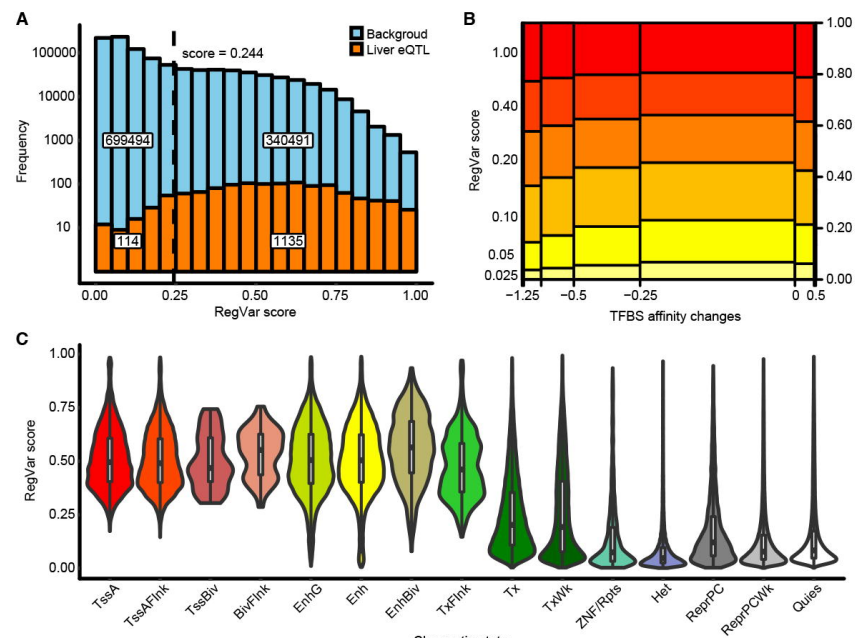

Chromatin state 
A

Tissue-shared regulatory variants

Tissue-specific regulatory variants
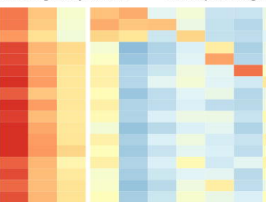

Non-functional vatiants

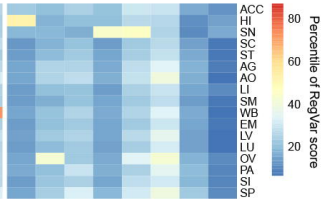

B

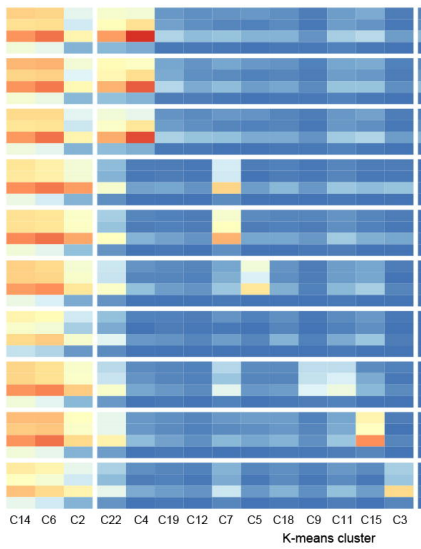

Dnase in $\mathrm{ACC}$ $\mathrm{H} 3 \mathrm{~K} 27 \mathrm{ac}$ in $\mathrm{ACC}$ $\mathrm{H} 3 \mathrm{~K} 4 \mathrm{me} 1$ in $\mathrm{ACC}$ H3K4me3 in ACC

Dnase in $\mathrm{HI}$

$\mathrm{H} 3 \mathrm{~K} 27 \mathrm{ac}$ in $\mathrm{HI}$ H3K4me 1 in $\mathrm{HI}$ $\mathrm{H} 3 \mathrm{~K} 4 \mathrm{me} 3 \mathrm{in} \mathrm{HI}$

Dnase in SN

$\mathrm{H} 3 \mathrm{~K} 27 \mathrm{ac}$ in $\mathrm{SN}$ H3K4me 1 in SN $\mathrm{H} 3 \mathrm{~K} 4 \mathrm{me}$ 3 in SN

Dnase in $\mathrm{SC}$ $\mathrm{H} 3 \mathrm{~K} 27 \mathrm{ac}$ in $\mathrm{SC}$ H3K 4 me 1 in SC H3K4me3 in SC

Dnase in ST H3K27 ac in ST H3K4me 1 in ST H3K4me3 in ST

Dnase in SG

$\mathrm{H} 3 \mathrm{~K} 27 \mathrm{ac}$ in $\mathrm{AG}$ H3K 4 me 1 in $A G$ H3K4me 3 in $A G$

Dnase in $A O$

$\mathrm{H} 3 \mathrm{~K} 27 \mathrm{ac}$ in $\mathrm{AO}$ $\mathrm{H} 3 \mathrm{~K} 4 \mathrm{me} 1 \mathrm{in} \mathrm{AO}$ $\mathrm{H} 3 \mathrm{~K} 4 \mathrm{me} 3$ in $\mathrm{AO}$

Dnase in $\mathrm{LI}$

$\mathrm{H} 3 \mathrm{~K} 27 \mathrm{ac}$ in $\mathrm{LI}$ H3K4me 1 in L $\mathrm{H} 3 \mathrm{~K} 4 \mathrm{me} 3 \mathrm{in} \mathrm{L}$

Dnase in $\mathrm{SM}$ H3K27ac in SM H3K4me 1 in SM H3K4me 3 in SM

Dnase in WB H3K27ac in WB $\mathrm{H} 3 \mathrm{~K} 4 \mathrm{me} 1$ in WB H3K4me3 in WB

C1 $\mathrm{C} 8 \mathrm{C} 10 \mathrm{C} 13 \mathrm{C} 16 \mathrm{C} 17 \quad \mathrm{C} 20 \quad \mathrm{C} 21$
0.8

0.6

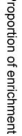

0.4 


\section{Random-variant}

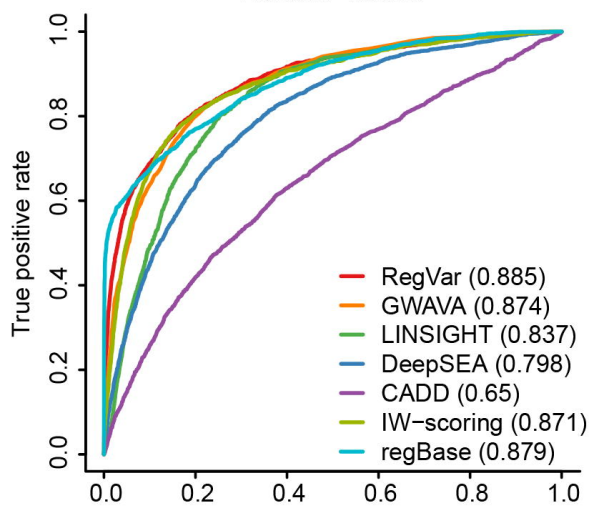

Distance-control-variant

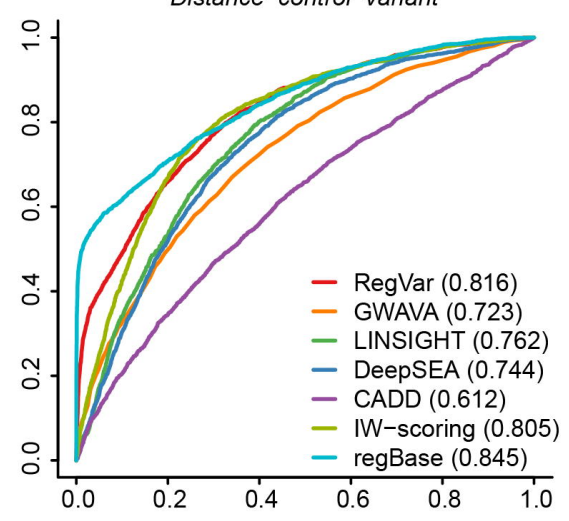

False positive rate

\section{Neighboring-variant}

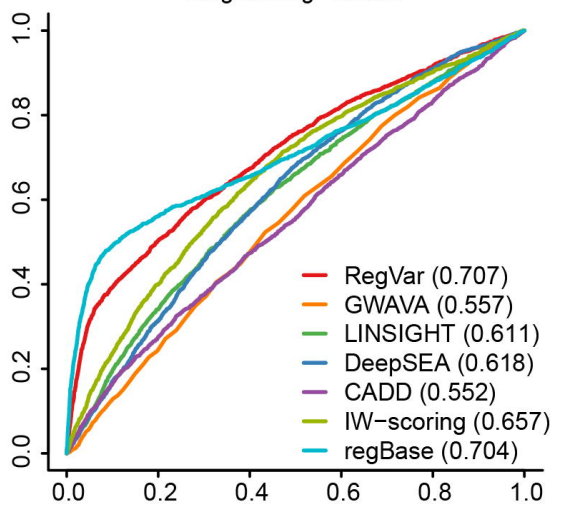


AUC of different sample sizes for skeletal muscle eQTL

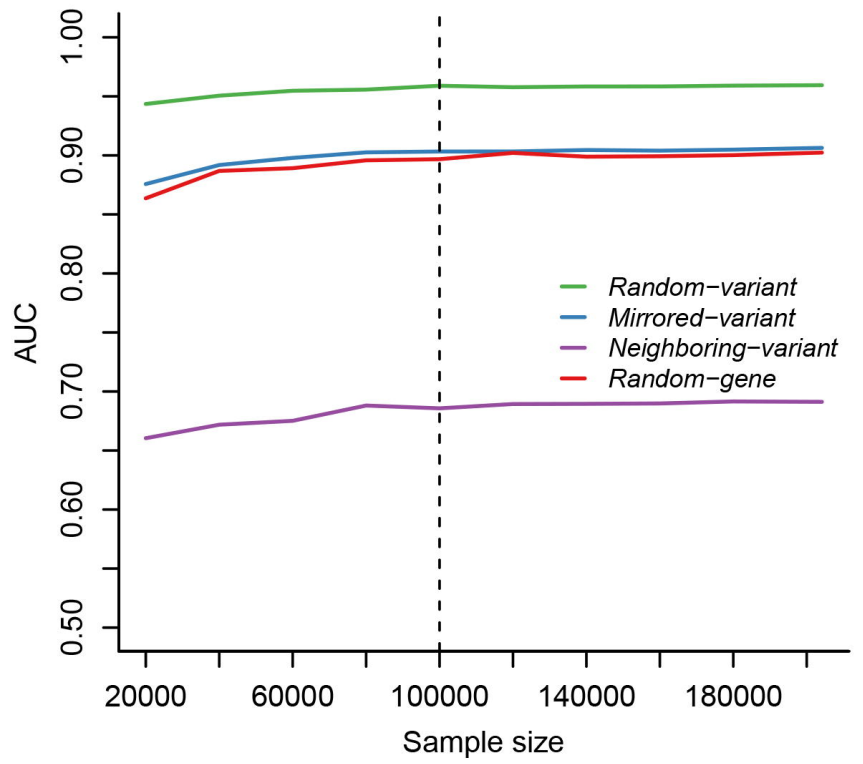




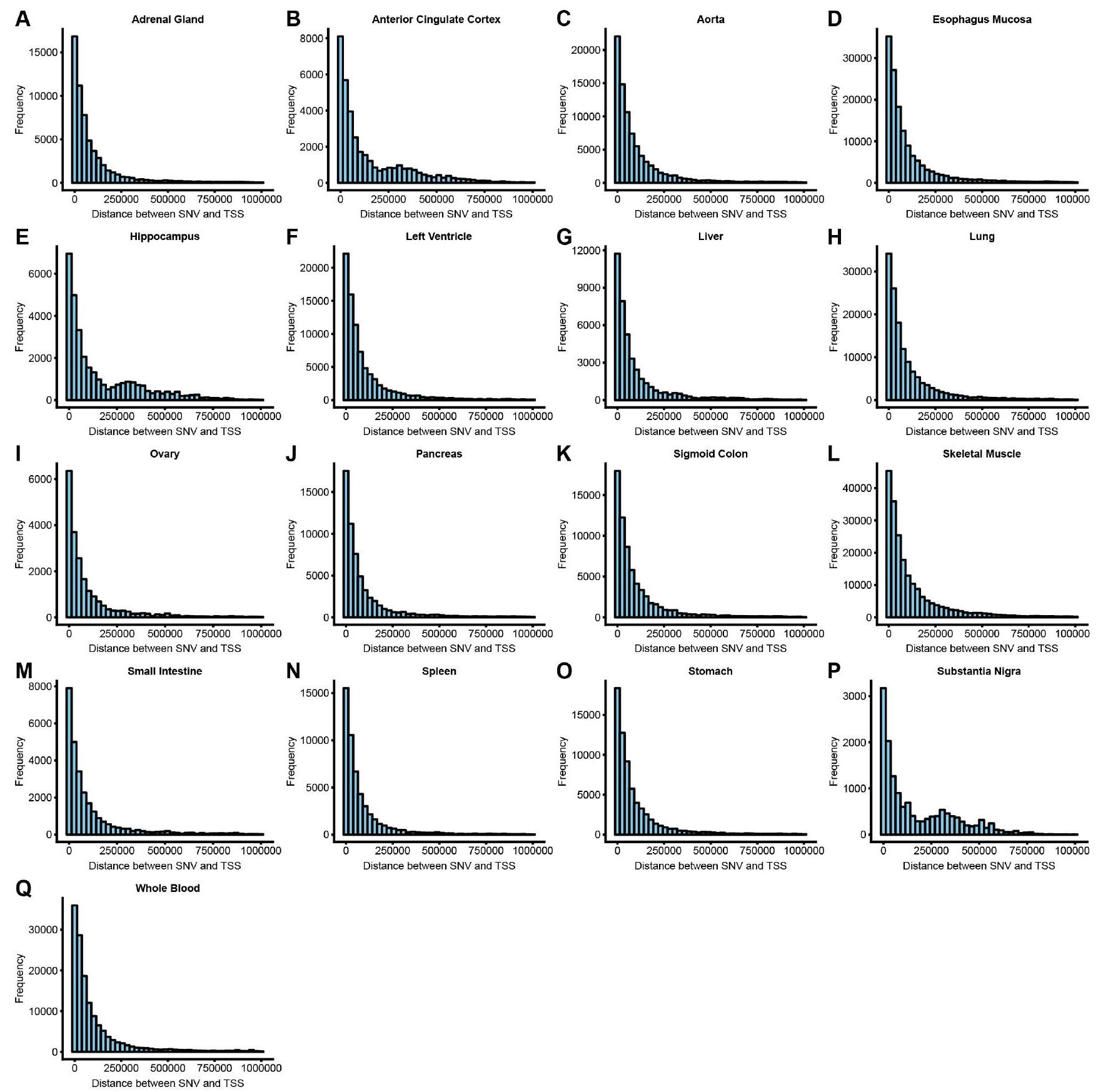




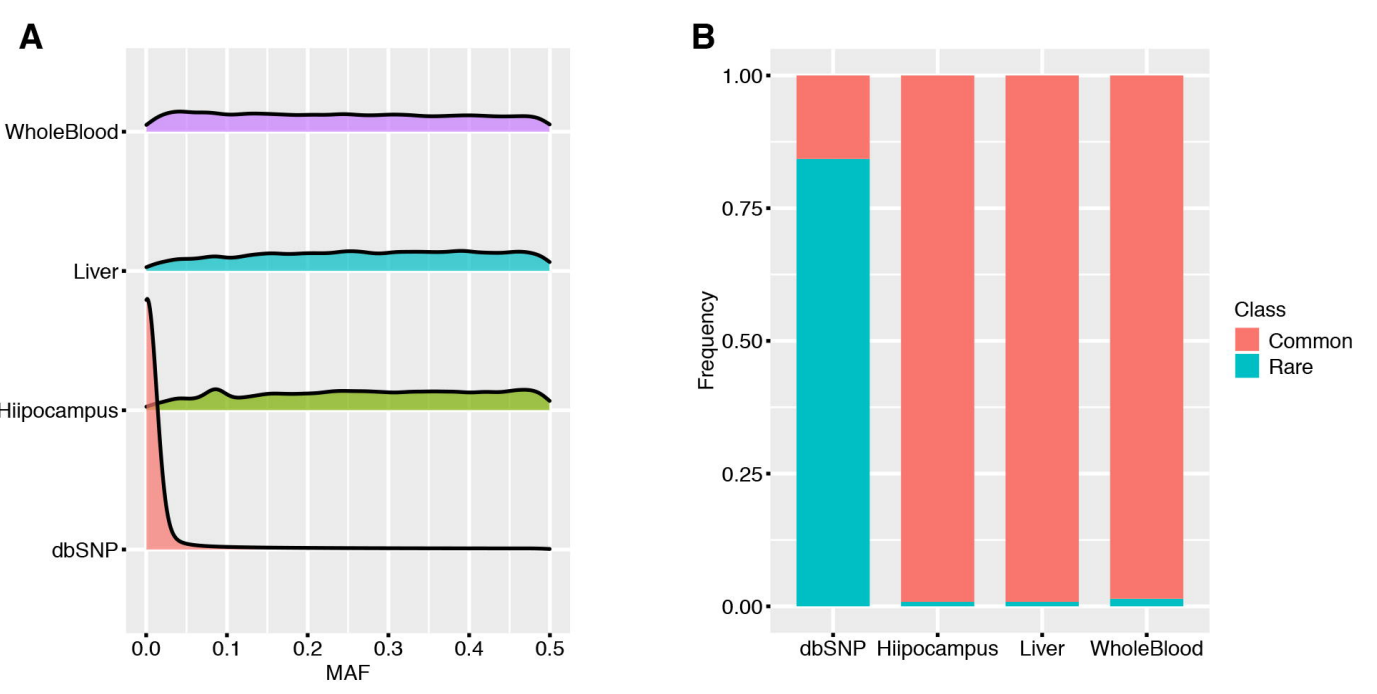


Original sample-full predictors $(0.919)$ Original sample-DHS predictor $(0.505)$

- Positive set filtered by DHS $(0.969)$

- Positive set filtered by ATAC (0.985)

\begin{tabular}{llllll}
\hline 0.0 & 0.2 & 0.4 & 0.6 & 0.8 & 1.0
\end{tabular}
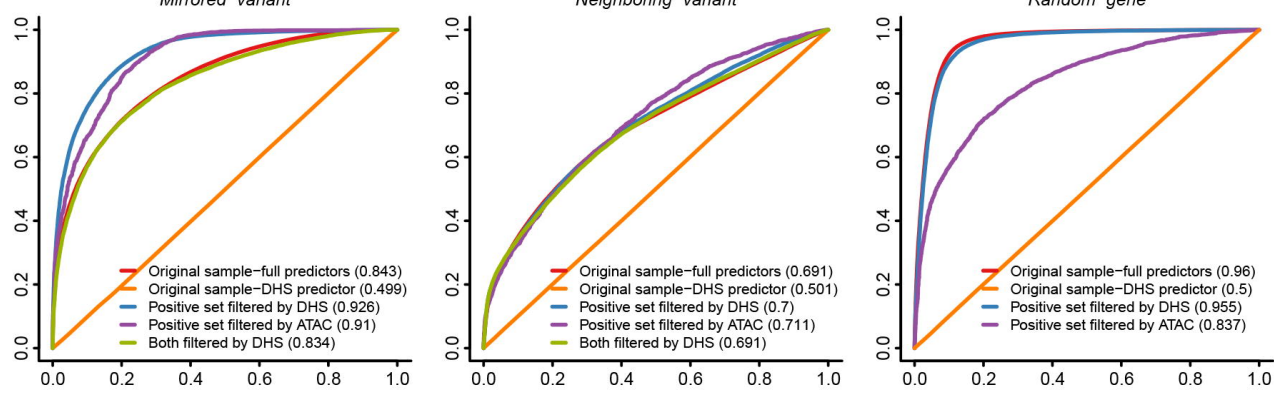

False positive rate 


\section{Random-variant}

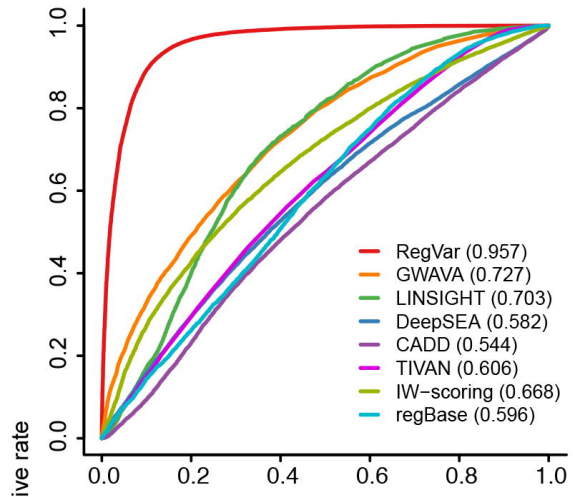

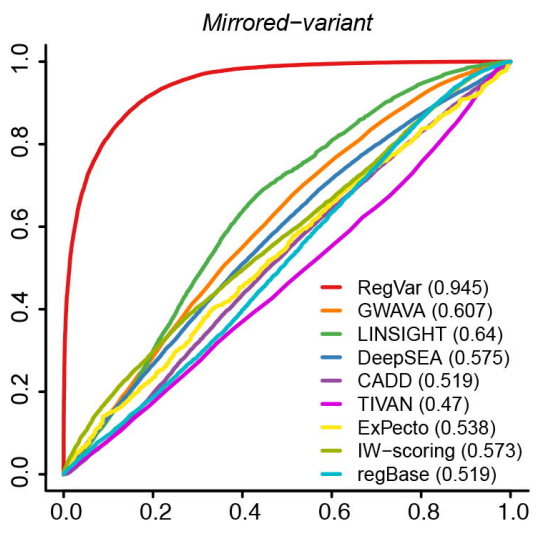

Neighboring-variant
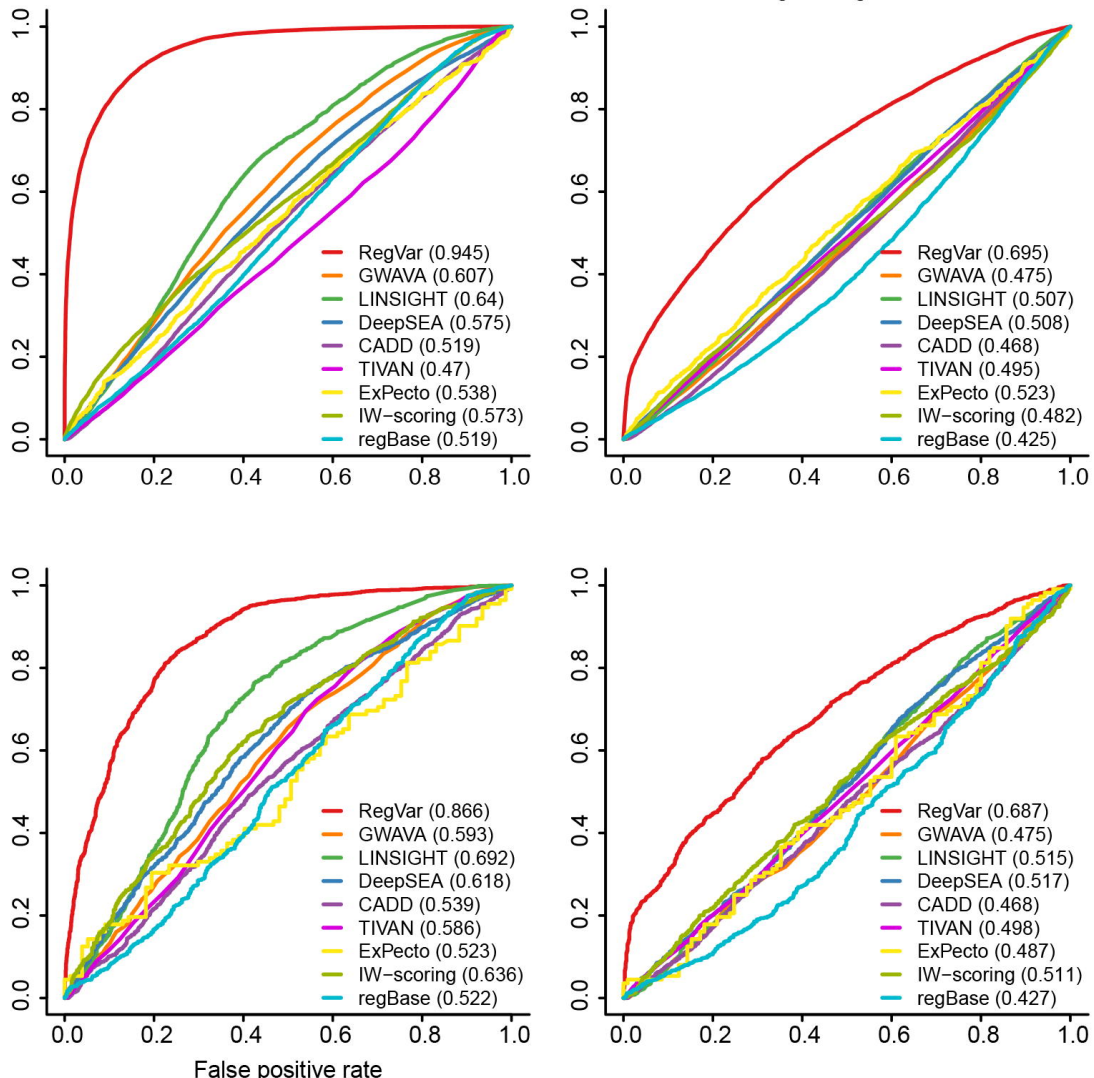

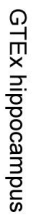

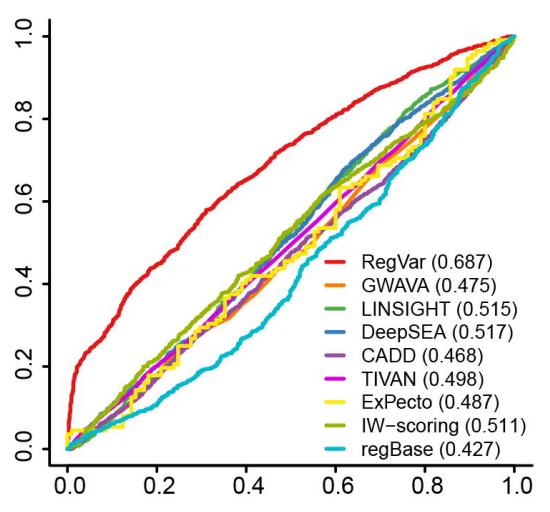

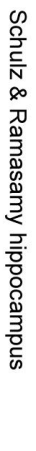




\section{Random-variant}

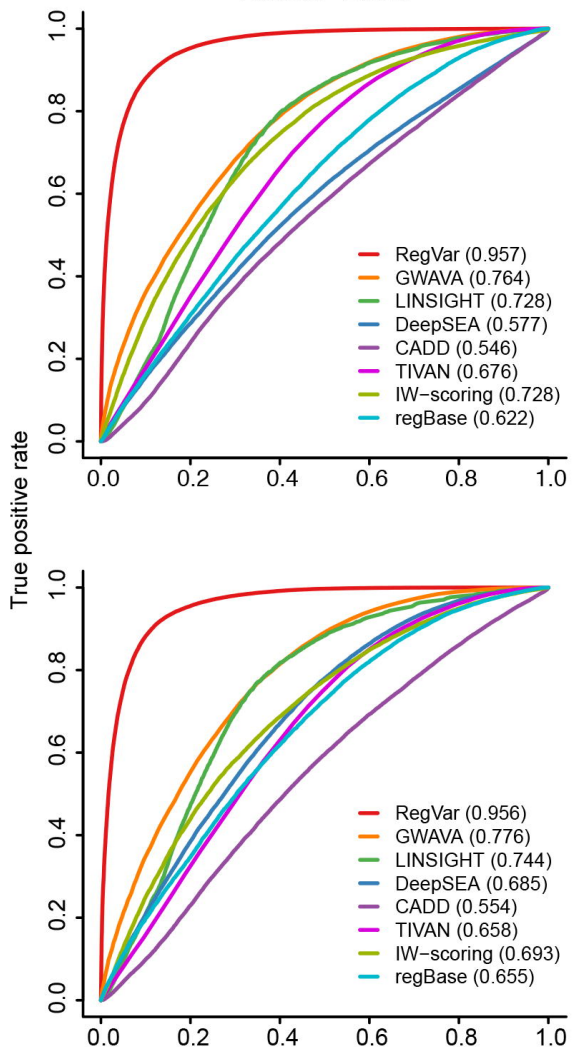

Mirrored-variant
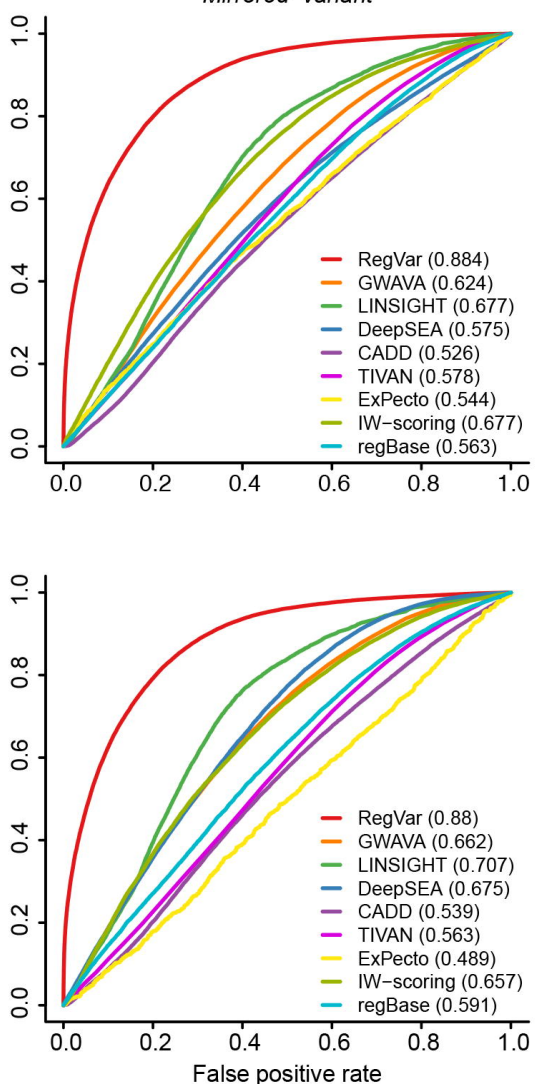

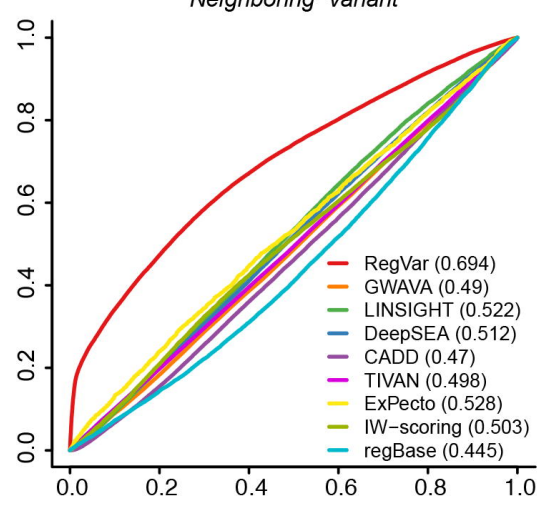

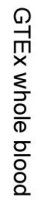

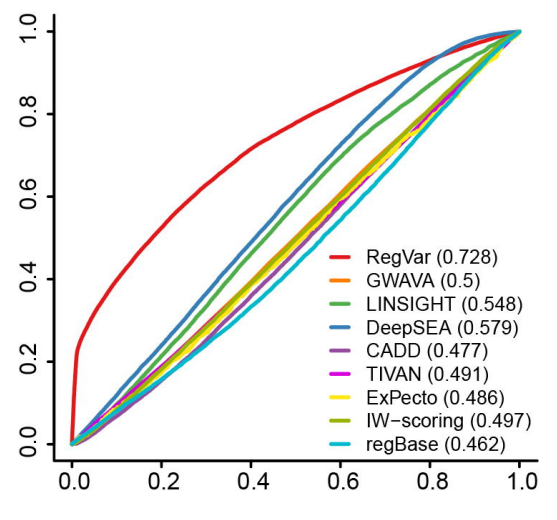




\section{Random-variant}
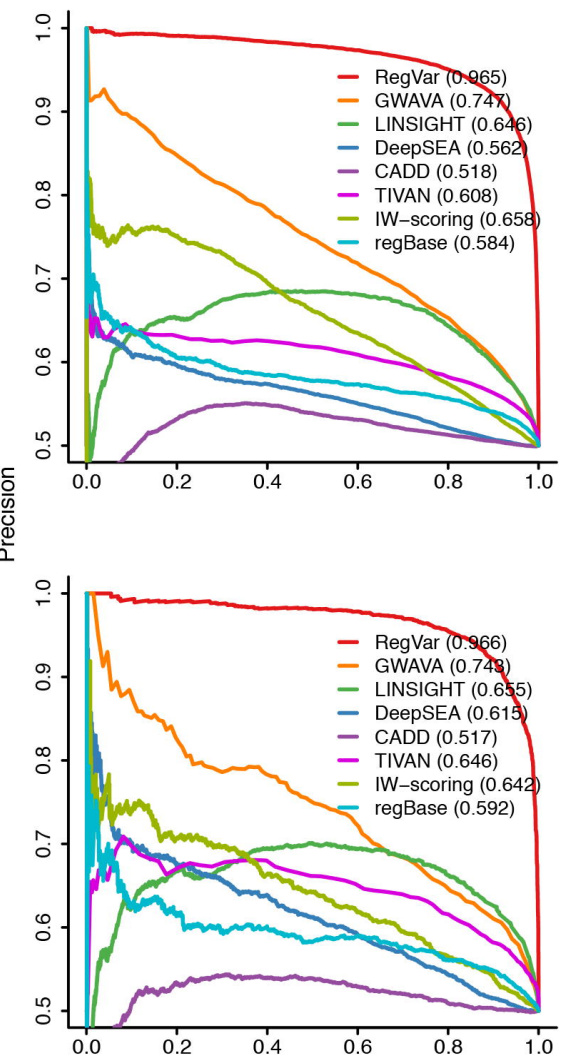

Mirrored-variant
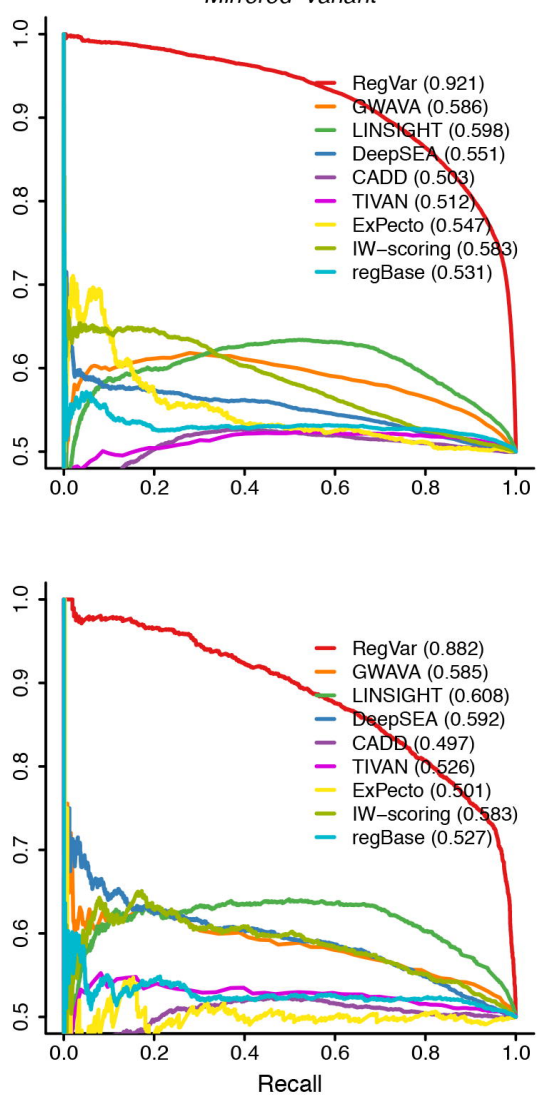

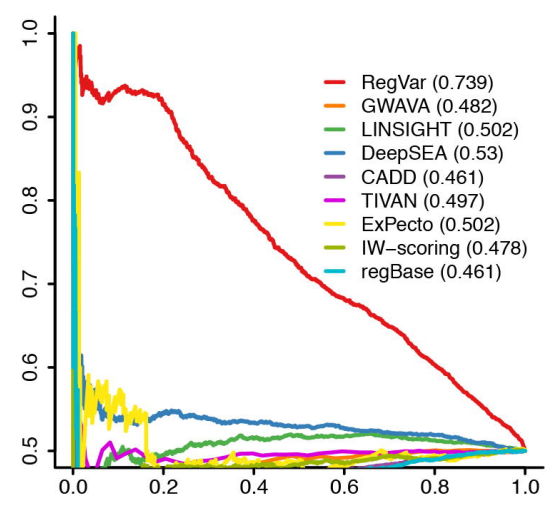

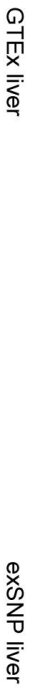
. 


\section{Random-variant}

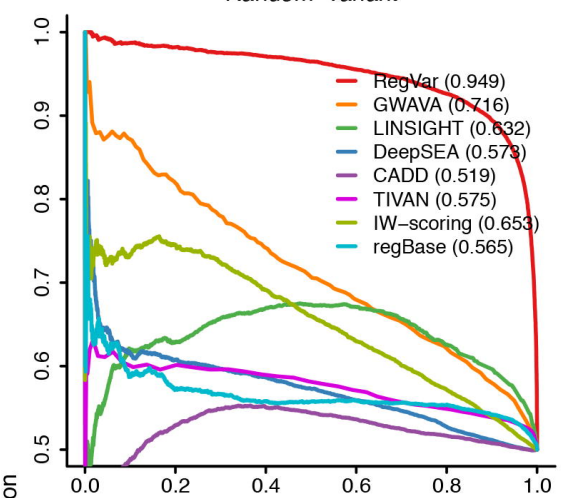

Mirrored-variant
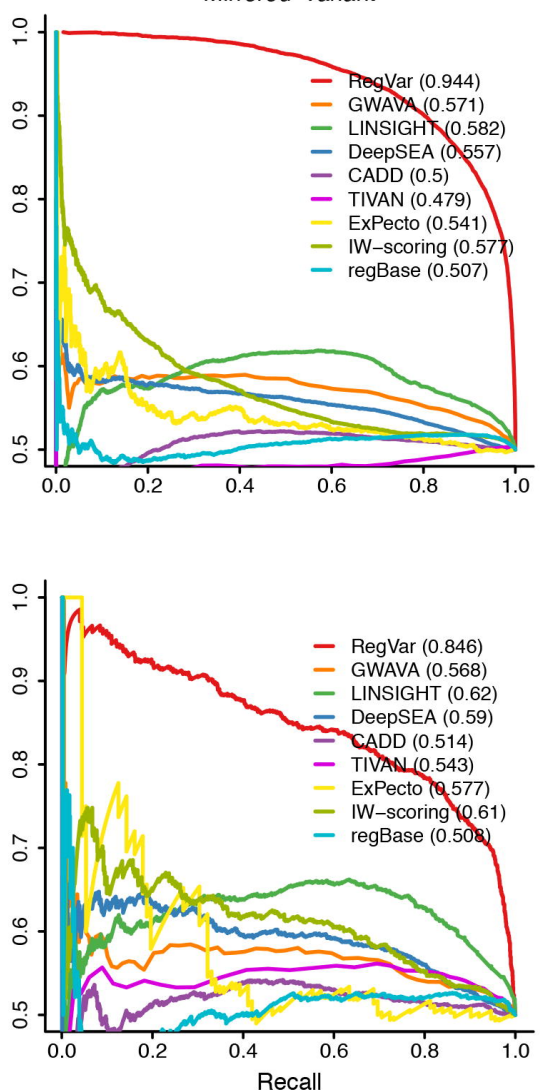
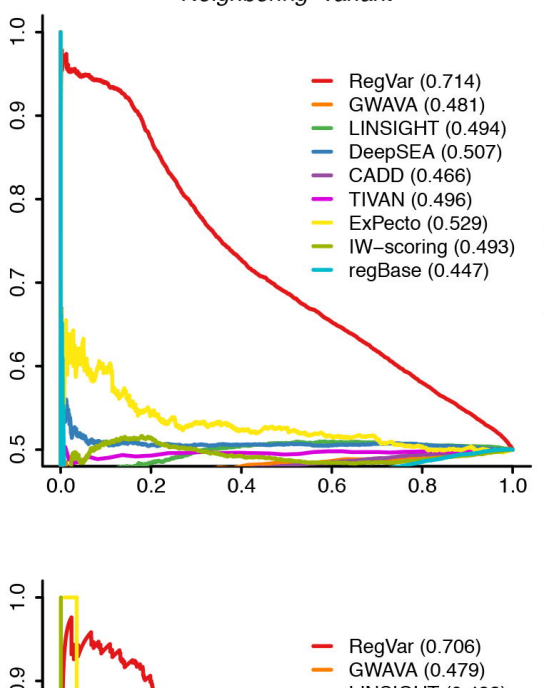

01 - LINSIGHT (0.492)

$-\operatorname{DeepSEA}(0.508)$
$-\operatorname{CADD}(0.471)$

$\stackrel{\infty}{\circ}$

- ExPecto (0.503)

$\begin{aligned}- & \text { IW-scoring }(0.51) \\ & - \text { regBase }(0.448)\end{aligned}$

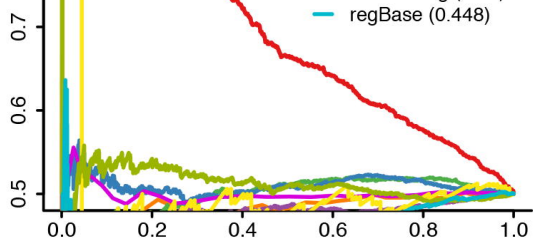

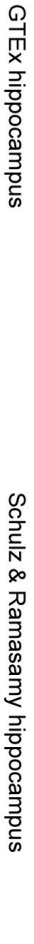


Random-variant

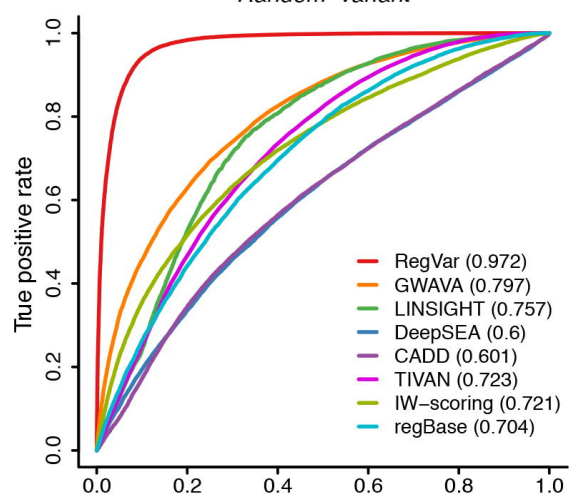

Mirrored-variant

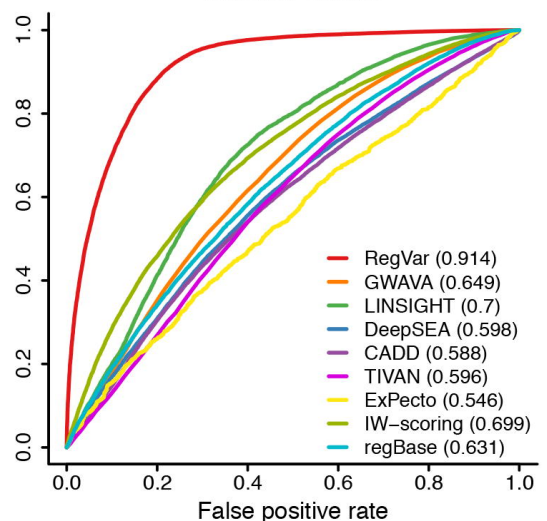

Neighboring-variant
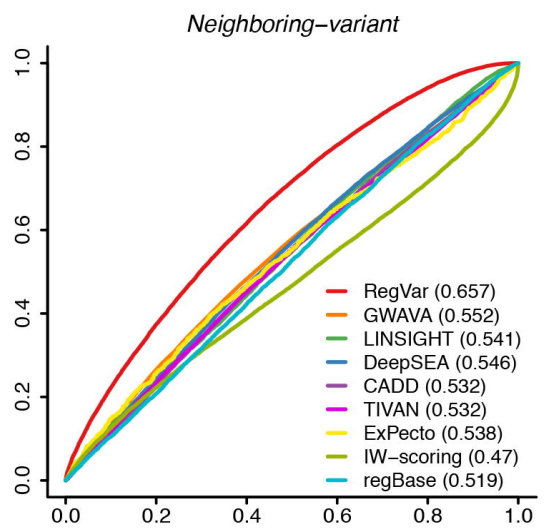

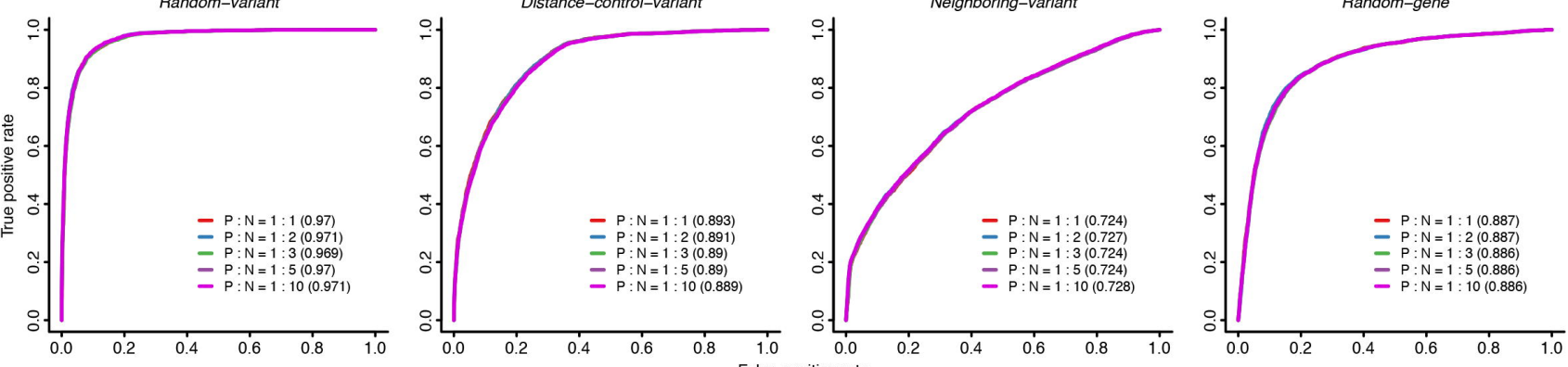


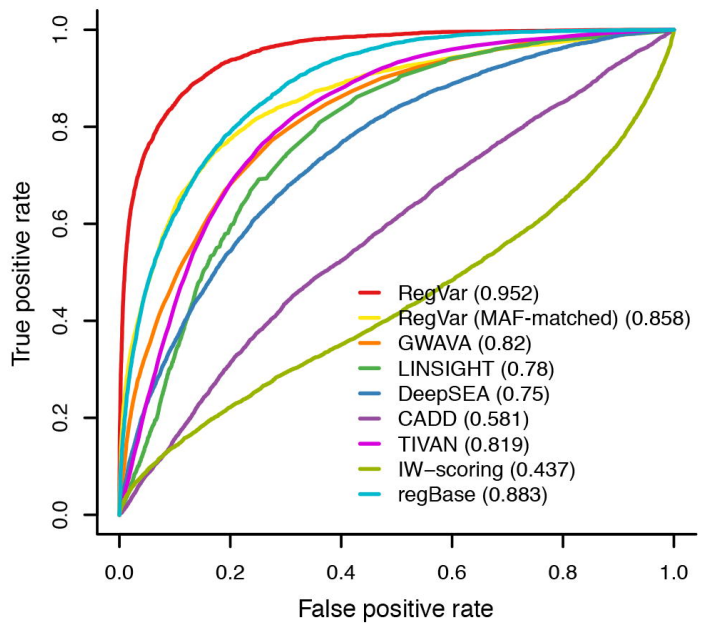




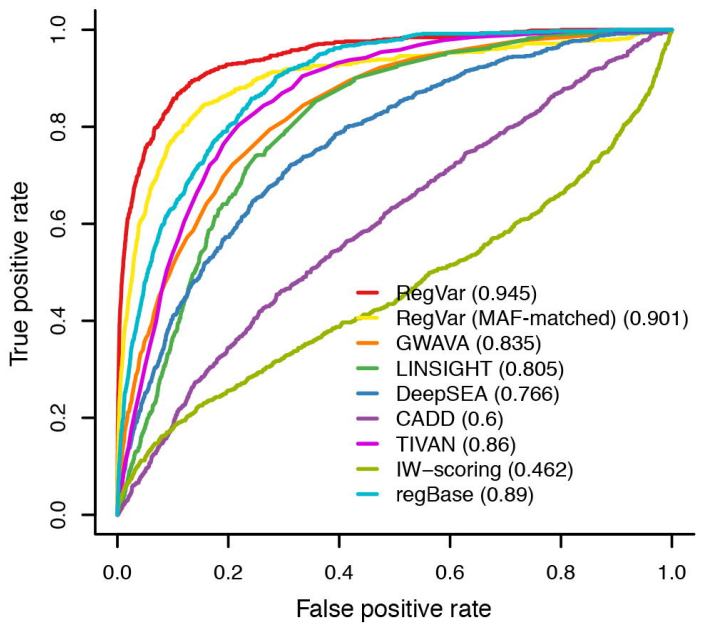




\section{Random-variant}

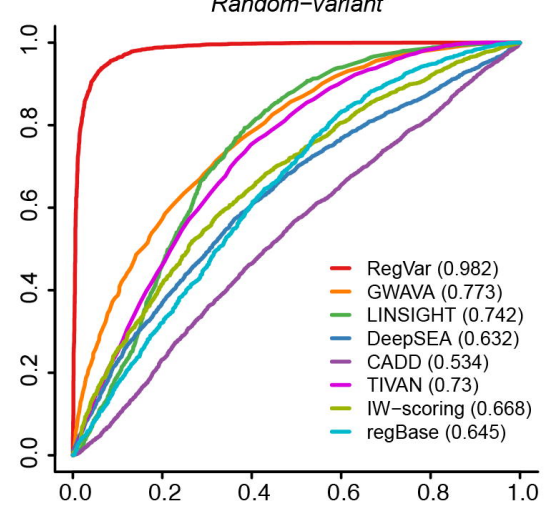

Mirrored-variant

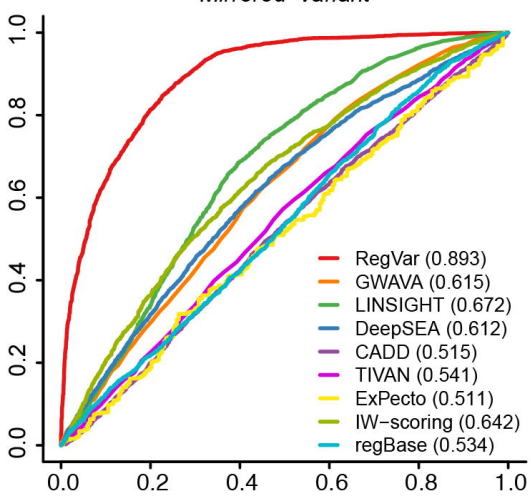

Neighboring-variant
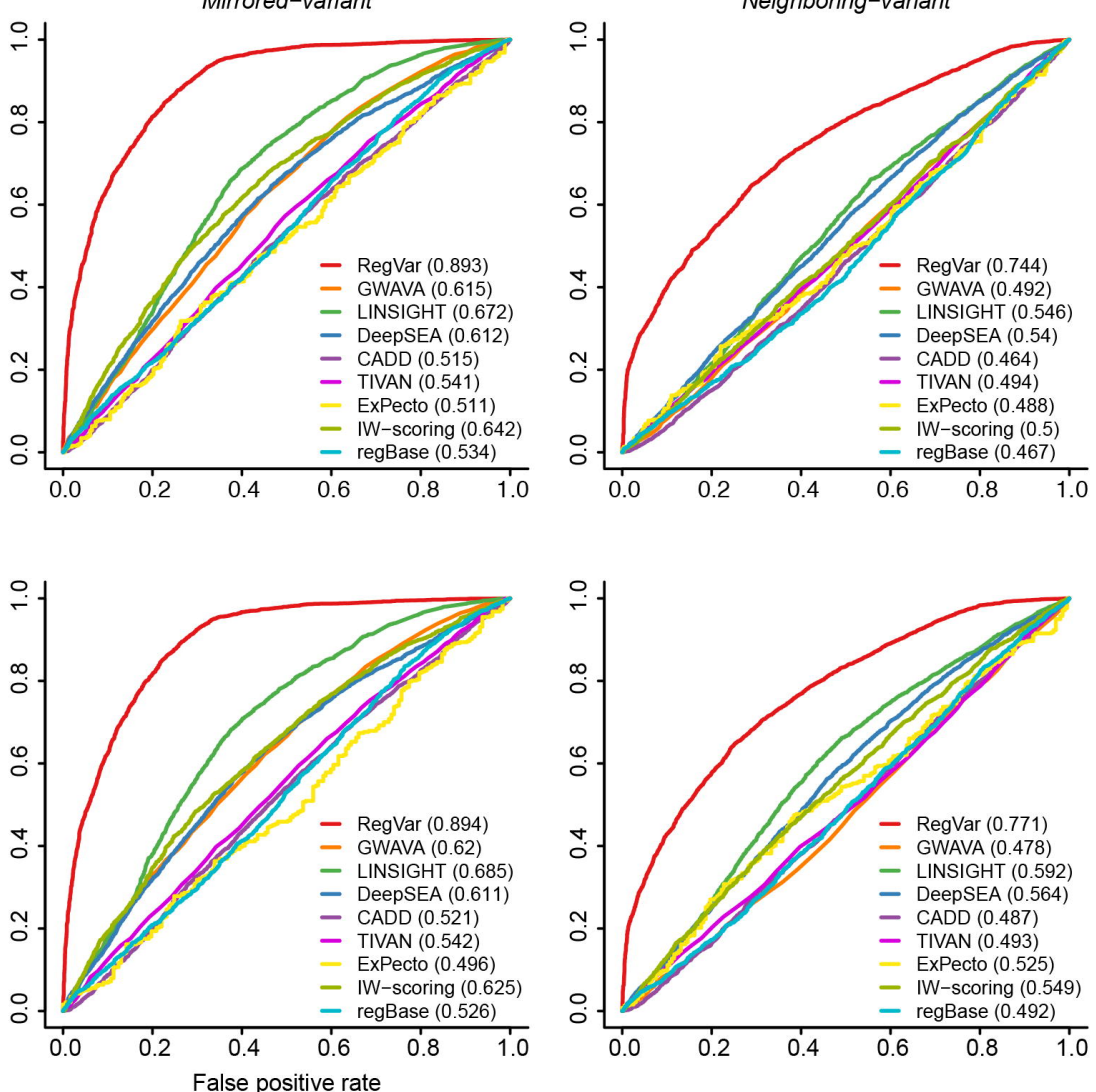

N

ठ N 중
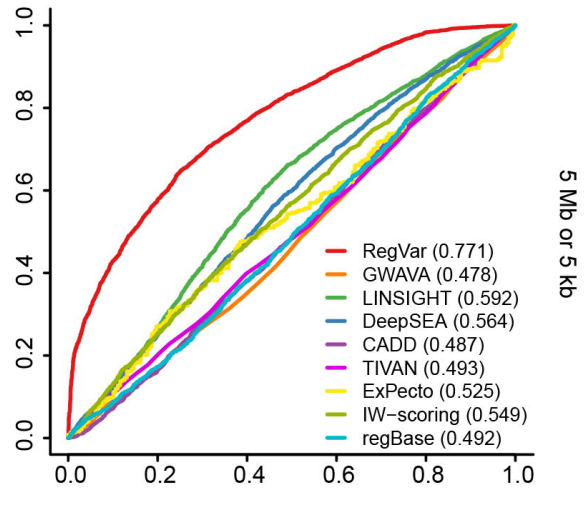

G
$\vdots$
$\sigma$
의
G
주 - GWAVA (0.784)

- LINSIGHT (0.756)

- DeepSEA (0.637)

- CADD (0.548)

- TIVAN (0.766)

- IW-scoring (0.678)

- regBase (0.669)

웅

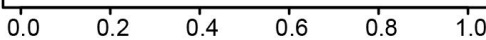




\section{A}

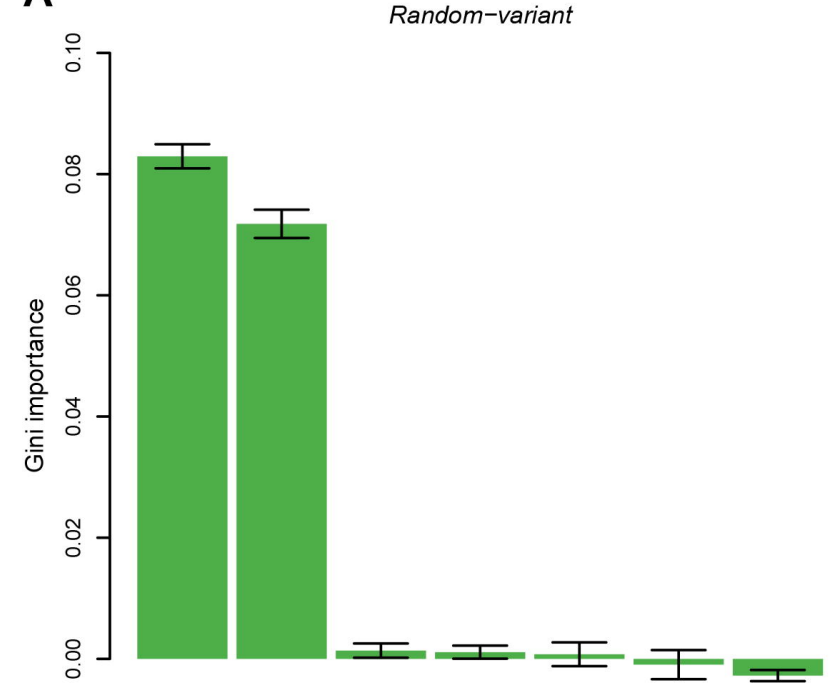

VarEpi Distance TSSEpi TSSSeq TSSEvo VarEvo VarSeq

C

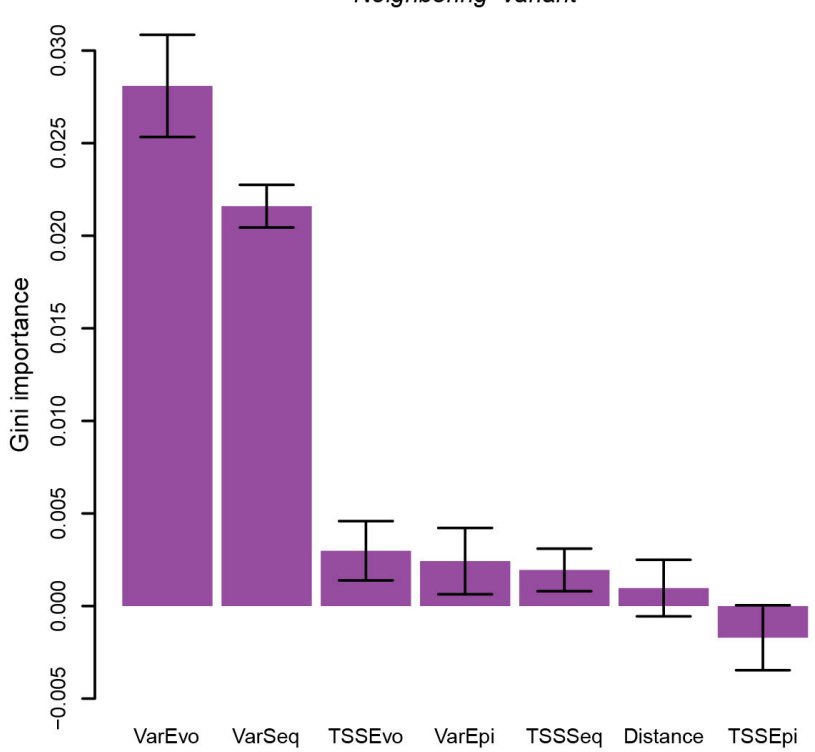

B
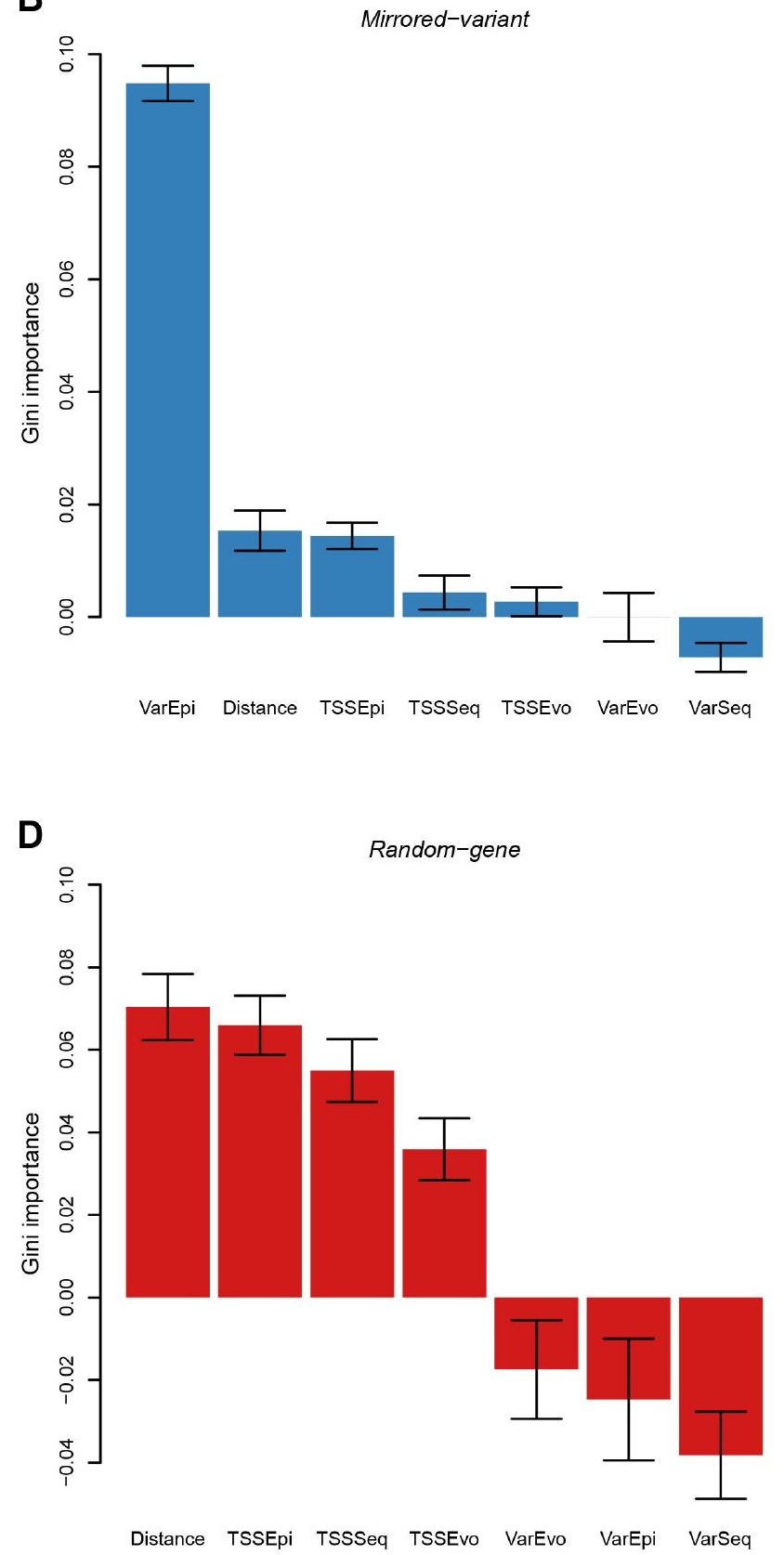
Random-variant

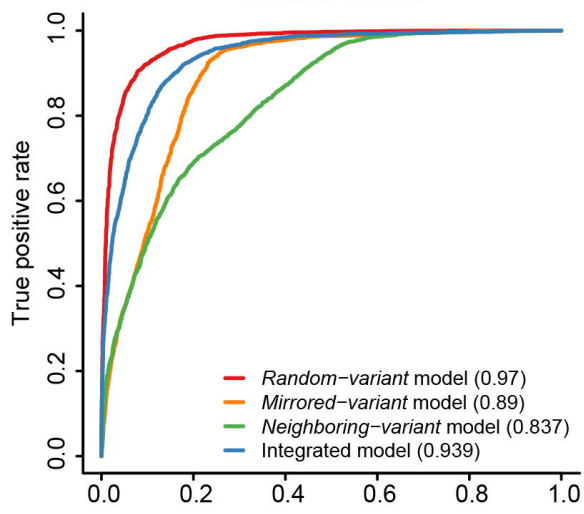

Mirrored-variant

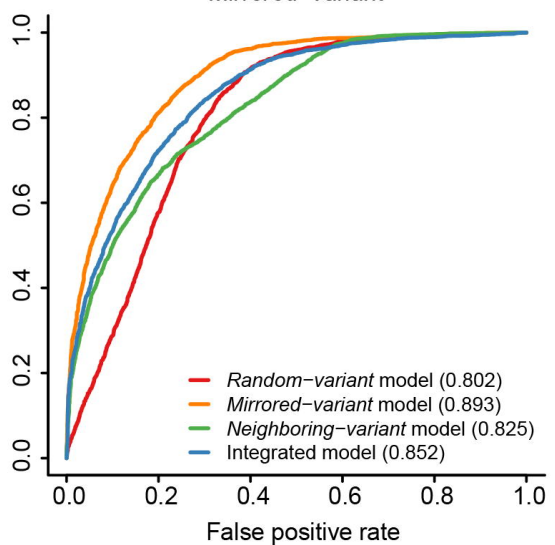

\section{Neighboring-variant}

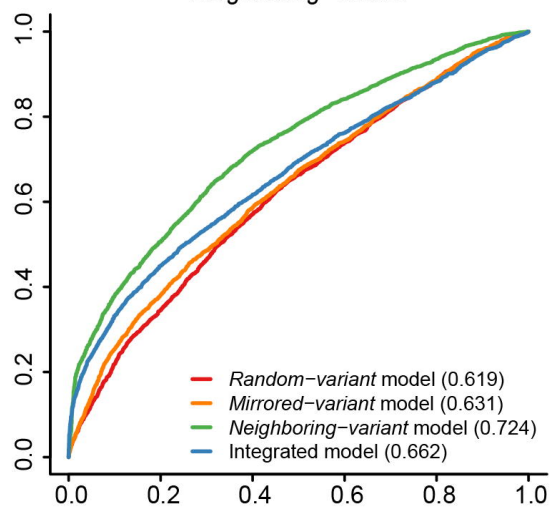




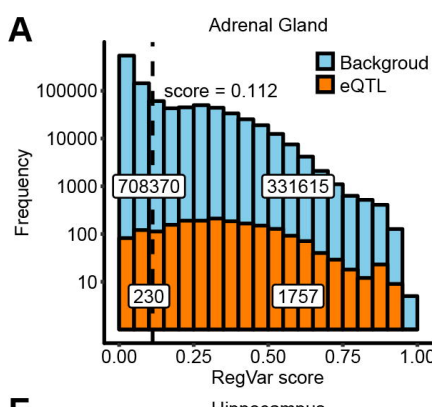

E

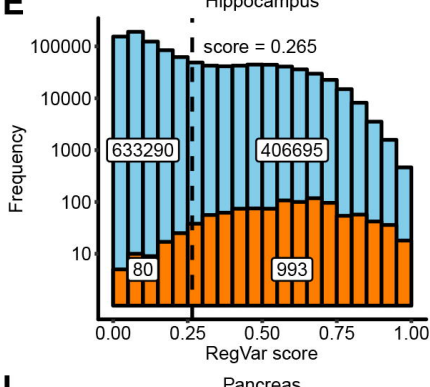

I

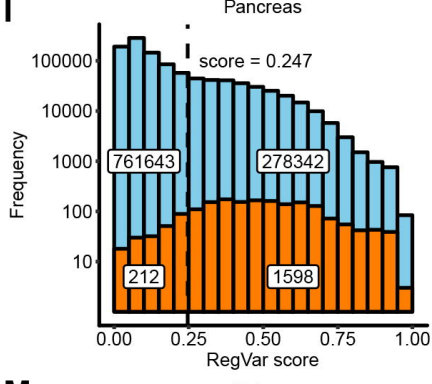

M

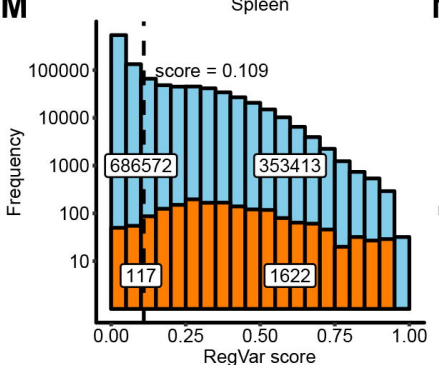

B Anterior Cingulate Cortex

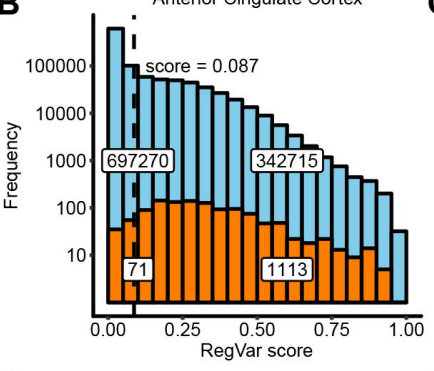

F
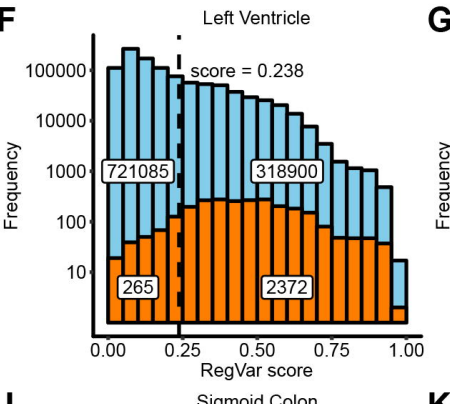

J

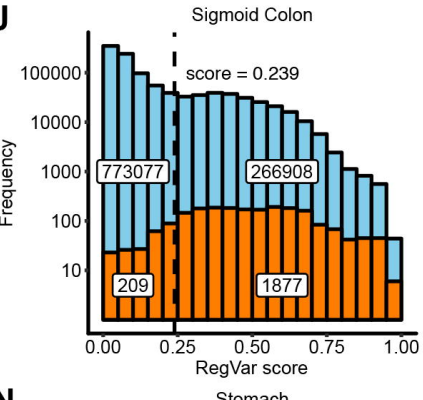

N

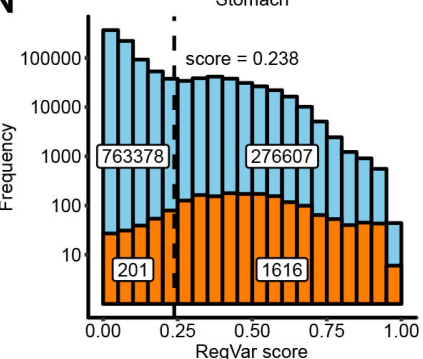

C

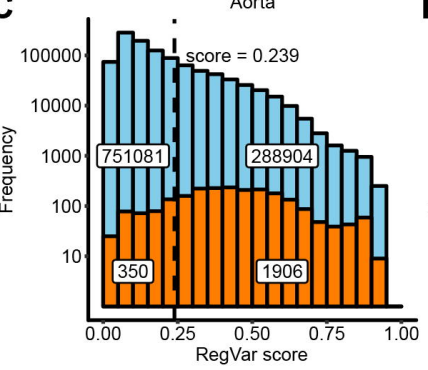

G

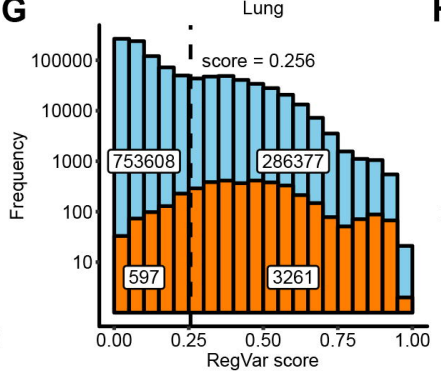

K Skeletal Muscle
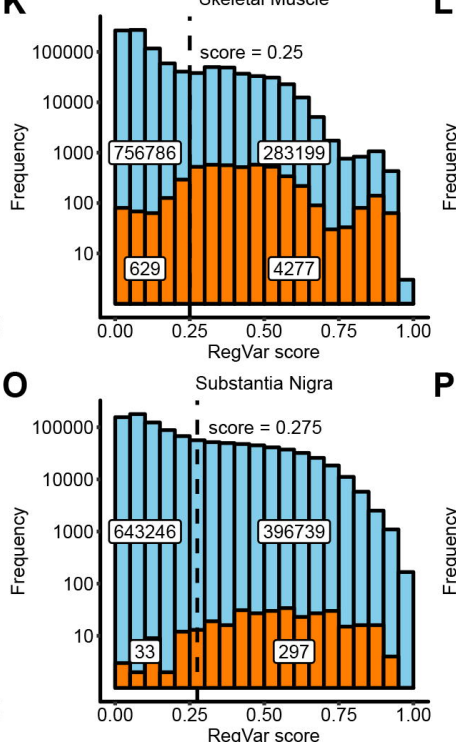

Esophagus Mucosa

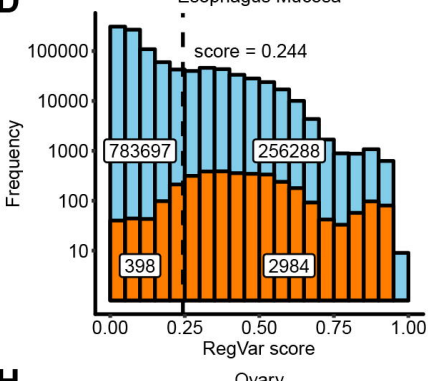

H

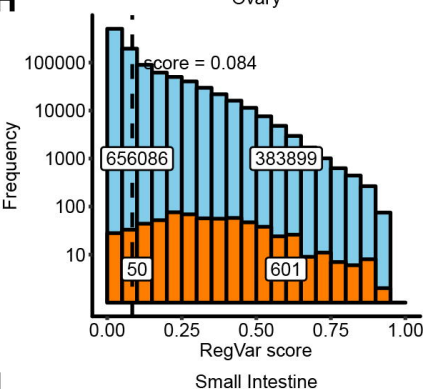

L Small Intestine

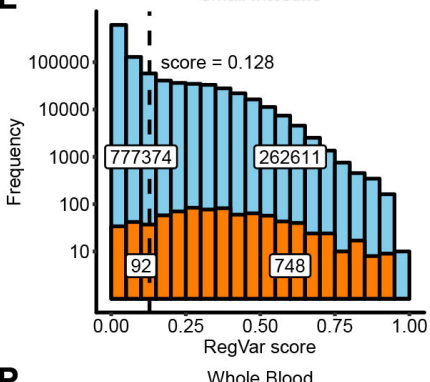

P Whole Blood

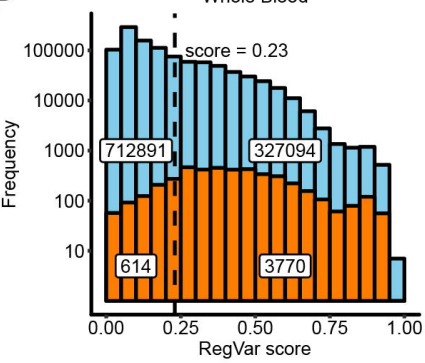


